\title{
Article \\ Generalized $p$-Convex Fuzzy-Interval-Valued Functions and Inequalities Based upon the Fuzzy-Order Relation
}

\author{
Muhammad Bilal Khan ${ }^{1, *(\mathbb{D} \text {, Savin Treanță }}{ }^{2, *}$ (D) and Hüseyin Budak ${ }^{3}$ \\ 1 Department of Mathematics, COMSATS University Islamabad, Islamabad 44000, Pakistan \\ 2 Department of Applied Mathematics, University Politehnica of Bucharest, 060042 Bucharest, Romania \\ 3 Department of Mathematics, Faculty of Science and Arts, Düzce University, Düzce 81620, Turkey; \\ huseyinbudak@duzce.edu.tr \\ * Correspondence: bilal42742@gmail.com (M.B.K.); savin.treanta@upb.ro (S.T.)
}

check for updates

Citation: Khan, M.B.; Treanță, S.; Budak, H. Generalized $p$-Convex Fuzzy-Interval-Valued Functions and Inequalities Based upon the Fuzzy-Order Relation. Fractal Fract. 2022, 6, 63. https://doi.org/10.3390/ fractalfract6020063

Academic Editor: Ravi P. Agarwal

Received: 30 November 2021

Accepted: 24 January 2022

Published: 26 January 2022

Publisher's Note: MDPI stays neutral with regard to jurisdictional claims in published maps and institutional affiliations.

Copyright: (c) 2022 by the authors. Licensee MDPI, Basel, Switzerland. This article is an open access article distributed under the terms and conditions of the Creative Commons Attribution (CC BY) license (https:// creativecommons.org/licenses/by/ $4.0 /)$.

\begin{abstract}
Convexity is crucial in obtaining many forms of inequalities. As a result, there is a significant link between convexity and integral inequality. Due to the significance of these concepts, the purpose of this study is to introduce a new class of generalized convex interval-valued functions called $(p, s)$-convex fuzzy interval-valued functions $((p, s)$-convex $F-I-V-F \mathrm{~s})$ in the second sense and to establish Hermite-Hadamard $(\mathrm{H}-\mathrm{H})$ type inequalities for $(p, s)$-convex $F-I-V-F$ s using fuzzy order relation. In addition, we demonstrate that our results include a large class of new and known inequalities for $(p, s)$-convex $F-I-V-F \mathrm{~s}$ and their variant forms as special instances. Furthermore, we give useful examples that demonstrate usefulness of the theory produced in this study. These findings and diverse approaches may pave the way for future research in fuzzy optimization, modeling, and interval-valued functions.
\end{abstract}

Keywords: $(p, s)$-convex fuzzy-interval-valued function; fuzzy Riemann integral; Jensen type inequality; Schur type inequality; Hermite-Hadamard type inequality; Hermite-Hadamard-Fejér type inequality

\section{Introduction}

A convex function has a convex set as its epigraph; therefore, the theory of inequality of convex functions falls under the umbrella of convexity. Nonetheless, it is a significant theory in and of itself, as it affects practically all fields of mathematics. The graphical analysis is most often the initial issue that necessitates the acquaintance with this theory. This is an opportunity to learn about the second derivative test of convexity, which is a useful tool for detecting convexity. The difficulty of identifying the extreme values of functions with many variables, as well as the application of Hessian as a higher dimensional generalization of the second derivative, follows. Holder, Jensen, and Minkowski all made early contributions to convex analysis. The next step is to go on to optimization issues in infinite dimensional spaces; however, despite the technological sophistication required to solve such problems, the fundamental concepts are quite similar to those underlying the one variable situation. Despite numerous applications, many contemporary difficulties in economics and engineering, the relevance of convex analysis is well recognized in optimization theory [1-3], and the idea of convexity no longer suffices.

Over the years, remarkable varieties of convexities, such as harmonic convexity [4], quasi convexity [5], Schur convexity [6], strong convexity [7,8], $p$-convexity [9], fuzzy convexity [10,11], fuzzy preinvexity [12] and generalized convexity [13], $p$-convexity [14] and so on, have been introduced to convex sets and convex functions. A fascinating field for research is the definition of convexity with an integral problem. Therefore, several authors have identified a great number of equalities or inequalities as applications of convex functions. The representative results include Gagliardo-Nirenberg-type inequality [15], Hardy-type inequality [16], Ostrowski-type inequality [17], Olsen-type inequality [18], 
and the most commonly known inequality of, namely, the $\mathrm{H}-\mathrm{H}$ inequality [19]. Similarly, many authors have devoted themselves to study the fractional integral inequalities for single-valued and interval-valued functions, see [20-28].

In ref. [29], the enormous research work fuzzy set and system has been dedicated on development of different fields, and it plays an important role in the study of a wide class problems arising in pure mathematics and applied sciences including operation research, computer science, managements sciences, artificial intelligence, control engineering and decision sciences. Recently, fuzzy interval analysis and fuzzy interval-valued differential equations have been put forward to deal the ambiguity originate by insufficient data in some mathematical or computer models that determine real-world phenomena [30-40]. There are some integrals to deal with fuzzy-interval-valued functions (in short, $F-I-V-F \mathrm{~s}$ ), where the integrands are F-I-V-Fs. For instance, Osuna-Gomez et al. [41], and Costa et al. [42] constructed Jensen's integral inequality for $F-I-V-F$ s through a Kulisch-Miranker order relation, see [43]. By using the same approach, Costa and Roman-Flores also presented Minkowski and Beckenbach's inequalities, where the integrands are $F-I-V-F$ s. This paper is motivated by [42-44] and especially by Costa et al. [45] because they established a relation between elements of fuzzy-interval space and interval space, and introduced level-wise fuzzy order relation on fuzzy-interval space through a Kulisch-Miranker order relation defined on interval space. For more information related to fuzzy interval calculus and generalized convex $F-I-V-F s$, see [46-61].

Inspired by the ongoing research work, the new class of generalized convex $F-I-V-F s$ is introduced, which is known as $(p, s)$-convex F-I-V-Fs. With the help of this class and fuzzy Riemann integral operator, we introduce Jensen, Schur, and fuzzy interval $\mathrm{H}-\mathrm{H}$ type inequalities via fuzzy order relation. Moreover, we show that our results include a wide class of new and known inequalities for $(p, s)$-convex $F-I-V$ - $F$ s and their variant forms as special cases. Some useful examples are also presented to verify the validity of our main results.

\section{Definitions and Basic Results}

Let $\mathcal{K}_{C}$ and $\mathbb{F}_{C}(\mathbb{R})$ be the collection of all closed and bounded intervals, and fuzzy intervals of $\mathbb{R}$. We use $\mathcal{K}_{C}^{+}$to represent the set of all positive intervals. The collection of all Riemann integrable real-valued functions, Riemann integrable $I-V$-Fs and fuzzy Riemann integrable $F-I-V-F$ s over $[\mathrm{t}, \mathrm{s}]$ is denoted by $\mathcal{R}_{[\mathrm{t}, \mathrm{s}]}, \mathcal{I} \mathcal{R}_{[\mathrm{t}, \mathrm{s}]}$, and $\mathcal{F} \mathcal{R}_{([\mathrm{t}, \mathrm{s}])}$, respectively. For more conceptions on interval-valued functions and fuzzy interval-valued functions, see [36,42-44]. Moreover, we have:

The inclusion " $\subseteq$ " means that

$$
\xi \subseteq \eta \text { if and only if, }\left[\xi_{*}, \xi^{*}\right] \subseteq\left[\eta_{*}, \eta^{*}\right] \text {, if and only if } \eta_{*} \leq \xi_{*}, \xi^{*} \leq \eta^{*},
$$

for all $\left[\boldsymbol{r}_{*}, \boldsymbol{r}^{*}\right],\left[\eta_{*}, \eta^{*}\right] \in \mathcal{K}_{C}$.

Remark 1 ([43]). The relation " $\leq_{I}$ " defined on $\mathcal{K}_{C}$ by

$$
\left[\boldsymbol{r}_{*}, \boldsymbol{r}^{*}\right] \leq_{I}\left[\eta_{*}, \eta^{*}\right] \text { if and only if } \boldsymbol{r}_{*} \leq \eta_{*}, \boldsymbol{r}^{*} \leq \eta^{*},
$$

for all $\left[\boldsymbol{r}_{*}, \boldsymbol{r}^{*}\right],\left[\eta_{*}, \eta^{*}\right] \in \mathcal{K}_{C} ;$ it is an order relation.

Proposition 1 ([7]). Let $\mathbb{F}_{C}(\mathbb{R})$ be a set of fuzzy numbers. If $\xi, \omega \in \mathbb{F}_{C}(\mathbb{R})$, then relation

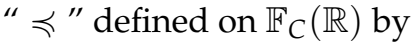

$$
\xi \preccurlyeq \omega \text { if and only if, }[\xi]^{\varphi} \leq_{I}[\omega]^{\varphi} \text {, for all } \varphi \in[0,1] \text {; }
$$

this relation is known as partial order relation.

Theorem 1 ([50]). Let $\mathfrak{U}:[\mathrm{t}, \mathrm{s}] \subset \mathbb{R} \rightarrow \mathbb{F}_{C}(\mathbb{R})$ be a $F-I-V-F$, whose $\varphi$-levels define the family of $I-V$-Fs $\mathfrak{U}_{\varphi}:[\mathrm{t}, \mathrm{s}] \subset \mathbb{R} \rightarrow \mathcal{K}_{C}$ are given by $\mathfrak{U}_{\varphi}(\varkappa)=\left[\mathfrak{U}_{*}(\varkappa, \varphi), \mathfrak{U}^{*}(\varkappa, \varphi)\right]$ for all $\in[\mathrm{t}, \mathrm{s}]$ 
and for all $\varphi \in(0,1]$. Then, $\mathfrak{U}$ is fuzzy Riemann integrable over $[t, s]$ if, and only if, $\mathfrak{U}_{*}(\varkappa, \varphi)$ and $\mathfrak{U}^{*}(\varkappa, \varphi)$ both are Riemann integrable over [t, s]. Moreover, if $\mathfrak{U}$ is fuzzy Riemann integrable over $[\mathrm{t}, \mathrm{s}]$, then

$$
\left((F R) \int_{\mathrm{t}}^{\mathrm{s}} \mathfrak{U}(\varkappa) d \varkappa\right)^{\varphi}=\left((R) \int_{\mathrm{t}}^{\mathrm{s}} \mathfrak{U}_{*}(\varkappa, \varphi) d \varkappa, \quad(R) \int_{\mathrm{t}}^{\mathrm{s}} \mathfrak{U}^{*}(\varkappa, \varphi) d \varkappa\right)=(I R) \int_{t}^{s} \mathfrak{U}_{\varphi}(\varkappa) d \varkappa,
$$

for all $\varphi \in(0,1]$.

Definition 1 ([10]). Let $K$ be a convex set. Then, $F-I-V-F \mathfrak{U}: K \rightarrow \mathbb{F}_{C}(\mathbb{R})$ is named as a convex $F-I-V-F$ on $K$ if the coming inequality

$$
\mathfrak{U}(\zeta+(1-\zeta) y) \preccurlyeq \zeta \mathfrak{U}(\varkappa) \widetilde{+}(1-\zeta) \mathfrak{U}(y)
$$

is valid for all , $y \in K, \zeta \in[0,1]$, where $\mathfrak{U}(\varkappa) \succcurlyeq \widetilde{0}$. If $(5)$ is reversed, then $\mathfrak{U}$ is named as a concave on $[t, s] . \mathfrak{U}$ is affine if and only if it is both a convex and concave function.

Definition 2. Let $K_{p}$ be a $p$-convex set and $s \in[0,1]$. Then, $F-I-V-F \mathfrak{U}: K_{p} \rightarrow \mathbb{F}_{C}(\mathbb{R})$ is named as a $(p, s)$-convex $F-I-V-F$ in the second sense on $K_{p}$ such that

$$
\mathfrak{U}\left(\left[\zeta \varkappa^{p}+(1-\zeta) y^{p}\right]^{\frac{1}{p}}\right) \preccurlyeq \zeta^{S} \mathfrak{U}(\varkappa) \widetilde{+}(1-\zeta)^{\mathcal{S}} \mathfrak{U}(y)
$$

for all $\varkappa, y \in K_{p}, \zeta \in[0,1]$, where $\mathfrak{U}(\varkappa) \succcurlyeq \widetilde{0}$. If (6) is reversed, then $\mathfrak{U}$ is named as a $(p, s)$-concave $F-I-V-F$ in the second sense on $[\mathrm{t}, \mathrm{s}] . \mathfrak{U}$ is $(p, s)$-affine if and only if it is both $(p, s)$-convex and $(p, s)$-concave $F-I-V-F$ in the second sense.

Remark 2. The $(p, s)$-convex $F-I-V$-Fs in the second sense have some very nice properties similar to convex $F-I-V-F$ :

- If we attempt to take $\mathfrak{U}$ as $(p, s)$-convex $F-I-V-F$, then we can obtain that $Y \mathfrak{U}$ is also $(p, s)$-convex $F-I-V-F$, for $Y \geq 0$;

- $\quad$ If we attempt to take both $\mathcal{F}$ and $\mathfrak{U}$ both as $(p, s)$-convex $F-I-V$ - $F$ s, then we can obtain that $\max (\mathcal{F}(\varkappa), \mathfrak{U}(\varkappa))$ is also a $(p, s)$-convex $F-I-V$-F.

We now discuss some new and known special cases of $(p, s)$-convex $F-I-V$-Fs in the second sense:

- If we attempt to take $s \equiv 1$, then from $(p, s)$-convex $F-I-V-F$, we achieve $p$-convex $F-I-V-F$, that is

$$
\mathfrak{U}\left(\left[\zeta \varkappa^{p}+(1-\zeta) y^{p}\right]^{\frac{1}{p}}\right) \preccurlyeq \zeta \mathfrak{U}(\varkappa) \widetilde{+}(1-\zeta) \mathfrak{U}(y), \forall \varkappa, y \in K, \zeta \in[0,1] .
$$

- If we attempt to take $p \equiv 1$, then from $(p, s)$-convex $F-I-V$ - $F$, we achieve $s$-convex $F-I-V-F$, see [13]; that is,

$$
\mathfrak{U}(\zeta \varkappa+(1-\zeta) y) \preccurlyeq \zeta^{s} \mathfrak{U}(\varkappa) \widetilde{+}(1-\zeta)^{s} \mathfrak{U}(y), \forall \varkappa, y \in K, \zeta \in[0,1], s \in[0,1] .
$$

- If we attempt to take $p \equiv 1$ and $s \equiv 1$, then from $(p, s)$-convex $F-I-V-F$, we achieve convex $F-I-V-F$, see $[13,36]$, that is

$$
\mathfrak{U}(\zeta \varkappa+(1-\zeta) y) \preccurlyeq \zeta \mathfrak{U}(\varkappa) \widetilde{+}(1-\zeta) \mathfrak{U}(y), \forall \varkappa, y \in K, \zeta \in[0,1] .
$$

Theorem 2. Let $K_{p}$ be $p$-convex set and $\mathfrak{U}: K_{p} \rightarrow \mathbb{F}_{C}(\mathbb{R})$ be a $F-I-V$-F, whose $\varphi$-levels define the family of IVFs $\mathfrak{U}_{\varphi}: K_{p} \subset \mathbb{R} \rightarrow \mathcal{K}_{C}{ }^{+} \subset \mathcal{K}_{C}$ are given by

$$
\mathfrak{U}_{\varphi}(\varkappa)=\left[\mathfrak{U}_{*}(\varkappa, \varphi), \mathfrak{U}^{*}(\varkappa, \varphi)\right],
$$


for all $\in K_{p}$ and for all $\varphi \in[0,1]$. Then, $\mathfrak{U}$ is $(p, s)$-convex F-I-V-F in the second sense on $K_{p}$, if and only if, for all $\varphi \in[0,1], \mathfrak{U}_{*}(\varkappa, \varphi)$ and $\mathfrak{U}^{*}(\varkappa, \varphi)$ both are $(p, s)$-convex functions in the second sense.

Proof. Assume that, for each $\varphi \in[0,1], \mathfrak{U}_{*}(\varkappa, \varphi)$ and $\mathfrak{U}^{*}(\varkappa, \varphi)$ are $(p, s)$-convex function in the second sense on $K_{p}$. Then, from Equation (6), we have

$$
\mathfrak{U}_{*}\left(\left[\zeta \varkappa^{p}+(1-\zeta) y^{p}\right]^{\frac{1}{p}}, \varphi\right) \leq \zeta^{s} \mathfrak{U}_{*}(\varkappa, \varphi)+(1-\zeta)^{s} \mathfrak{U}_{*}(y, \varphi), \forall \varkappa, y \in K_{p}, \zeta \in[0,1],
$$

and

$$
\mathfrak{U}^{*}\left(\left[\zeta \varkappa^{p}+(1-\zeta) y^{p}\right]^{\frac{1}{p}}, \varphi\right) \leq \zeta^{s} \mathfrak{U}^{*}(\varkappa, \varphi)+(1-\zeta)^{s} \mathfrak{U}^{*}(y, \varphi), \forall \varkappa, y \in K_{p}, \zeta \in[0,1] .
$$

Then, by Equation (10), we obtain

$$
\begin{gathered}
\mathfrak{U}_{\varphi}\left(\left[\zeta \varkappa^{p}+(1-\zeta) y^{p}\right]^{\frac{1}{p}}\right)=\left[\mathfrak{U}_{*}\left(\left[\zeta \varkappa^{p}+(1-\zeta) y^{p}\right]^{\frac{1}{p}}, \varphi\right), \mathfrak{U}^{*}\left(\left[\zeta \varkappa^{p}+(1-\zeta) y^{p}\right]^{\frac{1}{p}}, \varphi\right)\right], \\
\leq_{I}\left[\zeta^{\mathcal{S}} \mathfrak{U}_{*}(\varkappa, \varphi), \zeta^{s} \mathfrak{U}^{*}(\varkappa, \varphi)\right]+\left[(1-\zeta)^{\mathcal{S}} \mathfrak{U}_{*}(y, \varphi),(1-\zeta)^{\mathcal{S}} \mathfrak{U}^{*}(y, \varphi)\right],
\end{gathered}
$$

that is

$$
\mathfrak{U}\left(\left[\zeta \varkappa^{p}+(1-\zeta) y^{p}\right]^{\frac{1}{p}}\right) \preccurlyeq \zeta^{s} \mathfrak{U}(\varkappa) \widetilde{+}(1-\zeta)^{s} \mathfrak{U}(y), \forall \varkappa, y \in K_{p}, \zeta \in[0,1] .
$$

Hence, $\mathfrak{U}$ is $(p, s)$-convex $F-I-V-F$ in the second sense on $K_{p}$.

Conversely, let $\mathfrak{U}$ be $(p, s)$-convex $F-I-V-F$ in the second sense on $K_{p}$. Then, for all $\varkappa, y \in K_{p}$ and $\zeta \in[0,1]$, we have

$$
\mathfrak{U}\left(\left[\zeta \varkappa^{p}+(1-\zeta) y^{p}\right]^{\frac{1}{p}}\right) \preccurlyeq \zeta^{s} \mathfrak{U}(\varkappa) \widetilde{+}(1-\zeta)^{s} \mathfrak{U}(y) .
$$

Therefore, from Equation (10), we have

$$
\mathfrak{U}_{\varphi}\left(\left[\zeta \varkappa^{p}+(1-\zeta) y^{p}\right]^{\frac{1}{p}}\right)=\left[\mathfrak{U}_{*}\left(\left[\zeta \varkappa^{p}+(1-\zeta) y^{p}\right]^{\frac{1}{p}}, \varphi\right), \mathfrak{U}^{*}\left(\left[\zeta \varkappa^{p}+(1-\zeta) y^{p}\right]^{\frac{1}{p}}, \varphi\right)\right] .
$$

Again, from Equation (10), we obtain

$$
\zeta^{s} \mathfrak{U}_{\varphi}(\varkappa) \widetilde{+}(1-\zeta)^{s} \mathfrak{U}_{\varphi}(\varkappa)=\left[\zeta^{s} \mathfrak{U}_{*}(\varkappa, \varphi), \zeta^{s} \mathfrak{U}^{*}(\varkappa, \varphi)\right]+\left[(1-\zeta)^{s} \mathfrak{U}_{*}(y, \varphi),(1-\zeta)^{s} \mathfrak{U}^{*}(y, \varphi)\right],
$$

Then, by $(p, s)$-convexity in the second sense of $\mathfrak{U}$, we have

$$
\mathfrak{U}_{*}\left(\left[\zeta \varkappa^{p}+(1-\zeta) y^{p}\right]^{\frac{1}{p}}, \varphi\right) \leq \zeta^{s} \mathfrak{U}_{*}(\varkappa, \varphi)+(1-\zeta)^{s} \mathfrak{U}_{*}(y, \varphi),
$$

and

$$
\mathfrak{U}^{*}\left(\left[\zeta \varkappa^{p}+(1-\zeta) y^{p}\right]^{\frac{1}{p}}, \varphi\right) \leq \zeta^{s} \mathfrak{U}^{*}(\varkappa, \varphi)+(1-\zeta)^{s} \mathfrak{U}^{*}(y, \varphi),
$$

for each $\varphi \in[0,1]$. Hence, the result follows.

Remark 3. On the basis of Theorem 2, we consider the special situation as below:

- If we attempt to take $\mathfrak{U}_{*}(\varkappa, \varphi)=\mathfrak{U}^{*}(\varkappa, \varphi)$ with $\varphi=1$, then from Definition 2, we obtain the $(p, s)$-convex function, see [46];

- If we attempt to take $\mathfrak{U}_{*}(\varkappa, \varphi)=\mathfrak{U}^{*}(\varkappa, \varphi)$ with $\varphi=1$ and $s=1$, then from Definition 2 , we obtain the $p$-convex function, see [9]; 
- If we attempt to take $\mathfrak{U}_{*}(\varkappa, \varphi)=\mathfrak{U}^{*}(\varkappa, \varphi)$ with $\varphi=1, p=1$ and $s=0$, then from Definition 2, we obtain the $P$-function, see [47].

Example 1. We consider the $F-I-V-F \mathfrak{U}:[0,1] \rightarrow \mathbb{F}_{C}(\mathbb{R})$ defined by

$$
\mathfrak{U}(\varkappa)(\sigma)=\left\{\begin{array}{cc}
\frac{\sigma}{2 \varkappa^{p}} & \sigma \in\left[0,2 \varkappa^{p}\right] \\
\frac{4 \varkappa^{p}-\sigma}{2 \varkappa^{2}} & \sigma \in\left(2 \varkappa^{p}, 4 \varkappa^{p}\right] \\
0 & \text { otherwise, }
\end{array}\right.
$$

Then, for each $\varphi \in[0,1]$, we have $\mathfrak{U}_{\varphi}(\varkappa)=\left[2 \varphi \varkappa^{p},(4-2 \varphi) \varkappa^{p}\right]$. Since end point functions $\mathfrak{U}_{*}(\varkappa, \varphi)$ and $\mathfrak{U}^{*}(\varkappa, \varphi)$, both are $(p, s)$-convex functions in the second sense for each $\varphi \in[0,1]$ and $s \in[0,1]$. Hence, $\mathfrak{U}(\varkappa)$ is $(p, s)$-convex $F-I-V-F$ in the second sense.

\section{Discrete Inequalities for $(p, s)$-Convex F-I-V-F in the Second Sense}

In the following, we establish the following result:

Theorem 3. (Discrete Jensen type inequality for $(p, s)$-convex $F-I-V-F)$ Let $\omega_{j} \in \mathbb{R}^{+}$, $t_{j} \in[\mathrm{t}, \mathrm{s}],(j=1,2,3, \ldots, k, k \geq 2)$ and $\mathfrak{U}:[\mathrm{t}, \mathrm{s}] \rightarrow \mathbb{F}_{C}(\mathbb{R})$ be a $(p, s)$-convex $F-I-V-F$, whose $\varphi$-levels define the family of $I-V$-Fs $\mathfrak{U}_{\varphi}:[\mathrm{t}, \mathrm{s}] \subset \mathbb{R} \rightarrow \mathcal{K}_{C}+$ are given by $\mathfrak{U}_{\varphi}(\varkappa)=$ $\left[\mathfrak{U}_{*}(\varkappa, \varphi), \mathfrak{U}^{*}(\varkappa, \varphi)\right]$ for all $\in[\mathrm{t}, \mathrm{s}]$ and for all $\varphi \in[0,1]$, then

$$
\mathfrak{U}\left(\left[\frac{1}{W_{k}} \sum_{j=1}^{k} \omega_{j} \mathbf{t}_{j}^{p}\right]^{\frac{1}{p}}\right) \preccurlyeq \sum_{j}^{k}\left(\frac{\omega_{j}}{W_{k}}\right)^{s} \mathfrak{U}\left(\mathbf{t}_{j}\right),
$$

where $W_{k}=\sum_{j=1}^{k} \omega_{j}$. If $\mathfrak{U}$ is $(p, s)$-concave $F-I-V-F$, then inequality Equation (29) is reversed.

Proof. When $k=2$, then inequality Equation (12) is true. Considering that inequality Equation (29) is true for $k=n-1$, then

$$
\mathfrak{U}\left(\left[\frac{1}{W_{n-1}} \sum_{j=1}^{n-1} \omega_{j} \mathrm{t}_{j}^{p}\right]^{\frac{1}{p}}\right) \preccurlyeq \sum_{j=1}^{n-1}\left(\frac{\omega_{j}}{W_{n-1}}\right)^{s} \mathfrak{U}\left(\mathrm{t}_{j}\right)
$$

Now, let us prove that inequality (12) holds for $k=n$.

$$
\begin{aligned}
\mathfrak{U}\left(\left[\frac{1}{W_{n}} \sum_{j=1}^{n} \omega_{j} \mathbf{t}_{j}^{p}\right]^{\frac{1}{p}}\right) & \mathfrak{U}\left(\left[\frac{W_{n-2}}{W_{n}} \frac{1}{W_{n-2}} \sum_{j=1}^{n-2} \omega_{j} \mathbf{t}_{j}^{p}+\frac{\omega_{n-1}+\omega_{n}}{W_{n}}\left(\frac{\omega_{n-1}}{\omega_{n-1}+\omega_{n}} \mathbf{t}_{n-1}^{p}+\frac{\omega_{n}}{\omega_{n-1}+\omega_{n}} \mathbf{t}_{n}^{p}\right)\right]^{\frac{1}{p}}\right) .
\end{aligned}
$$

Therefore, for each $\varphi \in[0,1]$, we have

$$
\begin{aligned}
& \mathfrak{U}_{*}\left(\left[\frac{1}{W_{n}} \sum_{j=1}^{n} \omega_{j} \mathbf{t}_{j}\right]^{\frac{1}{p}}, \varphi\right) \\
& \mathfrak{U}^{*}\left(\left[\frac{1}{W_{n}} \sum_{j=1}^{n} \omega_{j} \mathbf{t}_{j}^{p}\right]^{\frac{1}{p}}, \varphi\right)
\end{aligned}
$$




$$
\begin{aligned}
& =\mathfrak{U}_{*}\left(\left[\frac{W_{n-2}}{W_{n}} \frac{1}{W_{n-2}} \sum_{j=1}^{n-2} \omega_{j} \mathbf{t}_{j}^{p}+\frac{\omega_{n-1}+\omega_{n}}{W_{n}}\left(\frac{\omega_{n-1}}{\omega_{n-1}+\omega_{n}} \mathbf{t}_{n-1}{ }^{p}+\frac{\omega_{n}}{\omega_{n-1}+\omega_{n}} \mathbf{t}_{n}^{p}\right)\right]^{\frac{1}{p}}, \varphi\right) \\
& =\mathfrak{U}^{*}\left(\left[\frac{W_{n-2}}{W_{n}} \frac{1}{W_{n-2}} \sum_{j=1}^{n-2} \omega_{j} \mathbf{t}_{j}^{p}+\frac{\omega_{n-1}+\omega_{n}}{W_{n}}\left(\frac{\omega_{n-1}}{\omega_{n-1}+\omega_{n}} \mathbf{t}_{n-1}{ }^{p}+\frac{\omega_{n}}{\omega_{n-1}+\omega_{n}} \mathbf{t}_{n}{ }^{p}\right)\right]^{\frac{1}{p}}, \varphi\right) \\
& \left.\leq \sum_{j=1}^{n-2}\left(\frac{\omega_{j}}{W_{n}}\right)^{S} \mathfrak{U}_{*}\left(\mathbf{t}_{j}, \varphi\right)+\left(\frac{\omega_{n-1}+\omega_{n}}{W_{n}}\right)^{S} \mathfrak{U}_{*}\left(\left[\frac{\omega_{n-1}}{\omega_{n-1}+\omega_{n}} \mathrm{t}_{n-1}{ }^{p}+\frac{\omega_{n}}{\omega_{n-1}+\omega_{n}} \mathbf{t}_{n}\right]^{p}\right]^{\frac{1}{p}}, \varphi\right) \\
& \leq \sum_{j=1}^{n-2}\left(\frac{\omega_{j}}{W_{n}}\right)^{\mathcal{S}} \mathfrak{U}^{*}\left(\mathbf{t}_{j}, \varphi\right)+\left(\frac{\omega_{n-1}+\omega_{n}}{W_{n}}\right)^{\mathcal{S}} \mathfrak{U}^{*}\left(\left[\frac{\omega_{n-1}}{\omega_{n-1}+\omega_{n}} \mathbf{t}_{n-1}{ }^{p}+\frac{\omega_{n}}{\omega_{n-1}+\omega_{n}} \mathbf{t}_{n}{ }^{p}\right]^{\frac{1}{p}}, \varphi\right) \\
& \leq \sum_{j=1}^{n-2}\left(\frac{\omega_{j}}{W_{n}}\right)^{S} \mathfrak{U}_{*}\left(\mathbf{t}_{j}, \varphi\right)+\left(\frac{\omega_{n-1}+\omega_{n}}{W_{n}}\right)^{S}\left[\left(\frac{\omega_{n-1}}{\omega_{n-1}+\omega_{n}}\right)^{S} \mathfrak{U}_{*}\left(\mathfrak{t}_{n-1}, \varphi\right)+\left(\frac{\omega_{n}}{\omega_{n-1}+\omega_{n}}\right)^{s} \mathfrak{U}_{*}\left(\mathfrak{t}_{n}, \varphi\right)\right] \\
& \leq \sum_{j=1}^{n-2}\left(\frac{\omega_{j}}{W_{n}}\right)^{S} \mathfrak{U}^{*}\left(\mathbf{t}_{j}, \varphi\right)+\left(\frac{\omega_{n-1}+\omega_{n}}{W_{n}}\right)^{S}\left[\left(\frac{\omega_{n-1}}{\omega_{n-1}+\omega_{n}}\right)^{S} \mathfrak{U}^{*}\left(\mathbf{t}_{n-1}, \varphi\right)+\left(\frac{\omega_{n}}{\omega_{n-1}+\omega_{n}}\right)^{S} \mathfrak{U}^{*}\left(\mathbf{t}_{n}, \varphi\right)\right] \\
& \leq \sum_{j=1}^{n-2}\left(\frac{\omega_{j}}{W_{n}}\right)^{s} \mathfrak{U}_{*}\left(\mathbf{t}_{j}, \varphi\right)+\left[\left(\frac{\omega_{n-1}}{W_{n}}\right)^{s} \mathfrak{U}_{*}\left(\mathbf{t}_{n-1}, \varphi\right)+\left(\frac{\omega_{n}}{W_{n}}\right)^{s} \mathfrak{U}_{*}\left(\mathbf{t}_{n}, \varphi\right)\right] \\
& \leq \sum_{j=1}^{n-2}\left(\frac{\omega_{j}}{W_{n}}\right)^{S} \mathfrak{U}^{*}\left(\mathfrak{t}_{j}, \varphi\right)+\left[\left(\frac{\omega_{n-1}}{W_{n}}\right)^{S} \mathfrak{U}^{*}\left(\mathfrak{t}_{n-1}, \varphi\right)+\left(\frac{\omega_{n}}{W_{n}}\right)^{S} \mathfrak{U}^{*}\left(\mathfrak{t}_{n}, \varphi\right)\right] \\
& =\sum_{j=1}^{n}\left(\frac{\omega_{j}}{W_{n}}\right)^{s} \mathfrak{U}_{*}\left(\mathbf{t}_{j}, \varphi\right) \\
& =\sum_{j=1}^{n}\left(\frac{\omega_{j}}{W_{n}}\right)^{\mathcal{S}} \mathfrak{U}^{*}\left(\mathbf{t}_{j}, \varphi\right) \text {. }
\end{aligned}
$$

From which, we have

$$
\begin{gathered}
{\left[\mathfrak{U}_{*}\left(\left[\frac{1}{W_{n}} \sum_{j=1}^{n} \omega_{j} \mathrm{t}_{j}\right]^{\frac{1}{p}}, \varphi\right), \mathfrak{U}^{*}\left(\left[\frac{1}{W_{n}} \sum_{j=1}^{n} \omega_{j} \mathrm{t}_{j}\right]^{\frac{1}{p}}, \varphi\right)\right]} \\
\leq_{I}\left[\sum_{j=1}^{n}\left(\frac{\omega_{j}}{W_{n}}\right)^{s} \mathfrak{U}_{*}\left(\mathrm{t}_{j}, \varphi\right), \sum_{j=1}^{n}\left(\frac{\omega_{j}}{W_{n}}\right)^{S} \mathfrak{U}^{*}\left(\mathrm{t}_{j}, \varphi\right)\right],
\end{gathered}
$$

that is,

$$
\mathfrak{U}\left(\left[\frac{1}{W_{n}} \sum_{j=1}^{n} \omega_{j} \mathbf{t}_{j}\right]^{\frac{1}{p}}\right) \preccurlyeq \sum_{j=1}^{n}\left(\frac{\omega_{j}}{W_{n}}\right)^{\mathcal{S}} \mathfrak{U}\left(\mathbf{t}_{j}\right),
$$

and the result follows.

If $\omega_{1}=\omega_{2}=\omega_{3}=\cdots=\omega_{k}=1$, then Theorem 3 reduces to the following result:

Corollary 1. Let $s \in[0,1] \mathrm{t}_{j} \in[\mathrm{t}, \mathrm{s}], .(j=1,2,3, \ldots, k, k \geq 2)$ and $\mathfrak{U}:[\mathrm{t}, \mathrm{s}] \rightarrow \mathbb{F}_{C}(\mathbb{R})$ be a $(p, s)$-convex $F-I-V-F$, whose $\varphi$-levels define the family of $I-V$-Fs $\mathfrak{U}_{\varphi}:[\mathrm{t}, \mathrm{s}] \subset \mathbb{R} \rightarrow \mathcal{K}_{C}{ }^{+}$ that are given by $\mathfrak{U}_{\varphi}(\varkappa)=\left[\mathfrak{U}_{*}(\varkappa, \varphi), \mathfrak{U}^{*}(\varkappa, \varphi)\right]$ for all $\in[\mathrm{t}, \mathrm{s}]$ and for all $\varphi \in[0,1]$; then,

$$
\mathfrak{U}\left(\left[\frac{1}{k} \sum_{j=1}^{k} \mathrm{t}_{j}{ }^{p}\right]^{\frac{1}{p}}\right) \preccurlyeq \sum_{J=1}^{k}\left(\frac{1}{k}\right)^{s} \mathfrak{U}\left(\mathbf{t}_{j}\right) .
$$

If $\mathfrak{U}$ is a $(p, s)$-concave $F-I-V-F$, then inequality Equation (13) is reversed.

The next Theorem 4 gives the Schur-type inequality for $(p, s)$-convex F-I-V-Fs.

Theorem 4. (Discrete Schur-type inequality for $(p, s)$-convex $F-I-V-F)$ Let $s \in[0,1]$ and $\mathfrak{U}:[\mathrm{t}, \mathrm{s}] \rightarrow \mathbb{F}_{C}(\mathbb{R})$ be a $(p, s)$-convex $F-I-V-F$, whose $\varphi$-levels define the family of IVFs $\mathfrak{U}_{\varphi}:[\mathrm{t}, \mathrm{s}] \subset \mathbb{R} \rightarrow \mathcal{K}_{C}{ }^{+}$are given by $\mathfrak{U}_{\varphi}(\varkappa)=\left[\mathfrak{U}_{*}(\varkappa, \varphi), \mathfrak{U}^{*}(\varkappa, \varphi)\right]$ for all $\in[\mathrm{t}, \mathrm{s}]$ and for all $\varphi \in[0,1]$. If $\mathrm{t}_{1}, \mathrm{t}_{2}, \mathrm{t}_{3} \in[\mathrm{t}, \mathrm{s}]$, such that $\mathrm{t}_{1}<\mathrm{t}_{2}<\mathrm{t}_{3}$ and $\mathrm{t}_{3}{ }^{p}-\mathrm{t}_{1}{ }^{p}, \mathrm{t}_{3}{ }^{p}-\mathrm{t}_{2}{ }^{p}$, $\mathrm{t}_{2}{ }^{p}-\mathrm{t}_{1}{ }^{p} \in[0,1]$, we have

$$
\left(\mathrm{t}_{3}{ }^{p}-\mathrm{t}_{1}^{p}\right)^{s} \mathfrak{U}\left(\mathrm{t}_{2}\right) \preccurlyeq\left(\mathrm{t}_{3}{ }^{p}-\mathrm{t}_{2}{ }^{p}\right)^{s} \mathfrak{U}\left(\mathrm{t}_{1}\right)+\left(\mathrm{t}_{2}{ }^{p}-\mathrm{t}_{1}^{p}\right)^{s} \mathfrak{U}\left(\mathrm{t}_{3}\right) .
$$


If $\mathfrak{U}$ is a $(p, s)$-concave $F-I-V-F$, then inequality Equation (14) is reversed.

Proof. Let $\mathrm{t}_{j}$ such that $L<\mathrm{t}_{j}\left\langle U(j=1,2,3, \ldots, k),\left(\mathrm{t}_{3} p-\mathrm{t}_{1}{ }^{p}\right)^{S}\right\rangle 0$. Then, by hypothesis, we have

$$
\left(\frac{\mathrm{t}_{3} p-\mathrm{t}_{2} p}{\mathrm{t}_{3} p-\mathrm{t}_{1} p}\right)^{s}=\frac{\left(\mathrm{t}_{3} p-\mathrm{t}_{2}\right)^{s}}{\left(\mathrm{t}_{3} p-\mathrm{t}_{1}\right)^{s}} \text { and }\left(\frac{\mathrm{t}_{2}{ }^{p}-\mathrm{t}_{1} p}{\mathrm{t}_{3} p-\mathrm{t}_{1}^{p}}\right)^{s}=\frac{\left(\mathrm{t}_{2}{ }^{p}-\mathrm{t}_{1}\right)^{s}}{\left(\mathrm{t}_{3} p-\mathrm{t}_{1}\right)^{s}} .
$$

Consider $\zeta=\frac{\mathrm{t}_{3}{ }^{p}-\mathrm{t}_{2}{ }^{p}}{\mathrm{t}_{3}{ }^{p}-\mathrm{t}_{1}{ }^{p}}$, then $\mathrm{t}_{2}{ }^{p}=\zeta \mathrm{t}_{1}{ }^{p}+(1-\zeta) \mathrm{t}_{3}{ }^{p}$. Since $\mathfrak{U}$ is a $(p, s)$-convex $F-I-V-F$ then, by hypothesis, we have

$$
\mathfrak{U}\left(\mathrm{t}_{2}\right) \preccurlyeq\left(\frac{\mathrm{t}_{3} p-\mathrm{t}_{2}{ }^{p}}{\mathrm{t}_{3} p-\mathrm{t}_{1}{ }^{p}}\right)^{s} \mathfrak{U}\left(\mathrm{t}_{1}\right)+\left(\frac{\mathrm{t}_{2}{ }^{p}-\mathrm{t}_{1} p}{\mathrm{t}_{3}{ }^{p}-\mathrm{t}_{1}{ }^{p}}\right)^{s} \mathfrak{U}\left(\mathrm{t}_{3}\right) .
$$

Therefore, for each $\varphi \in[0,1]$, we have

$$
\begin{aligned}
& \mathfrak{U}_{*}\left(\mathrm{t}_{2}, \varphi\right) \leq\left(\frac{\mathrm{t}_{3}{ }^{p}-\mathrm{t}_{2}{ }^{p}}{\mathrm{t}_{3}{ }^{p}-\mathrm{t}_{1}{ }^{p}}\right)^{\mathcal{S}} \mathfrak{U}_{*}\left(\mathrm{t}_{1}, \varphi\right)+\left(\frac{\mathrm{t}_{2}{ }^{p}-\mathrm{t}_{1}{ }^{p}}{\mathrm{t}_{3}{ }^{p}-\mathrm{t}_{1}{ }^{p}}\right)^{\mathcal{S}} \mathfrak{U}_{*}\left(\mathrm{t}_{3}, \varphi\right), \\
& \mathfrak{U}^{*}\left(\mathrm{t}_{2}, \varphi\right) \leq\left(\frac{\mathrm{t}_{3} p-\mathrm{t}_{2} p}{\mathfrak{t}_{3} p-\mathrm{t}_{1} p}\right)^{\mathcal{S}} \mathfrak{U}^{*}\left(\mathfrak{t}_{1}, \varphi\right)+\left(\frac{\mathrm{t}_{2} p-\mathfrak{t}_{1} p}{\mathfrak{t}_{3} p-t_{1} p}\right)^{\mathcal{S}} \mathfrak{U}^{*}\left(\mathfrak{t}_{3}, \varphi\right) \\
& =\frac{\left(\mathrm{t}_{3} p-\mathrm{t}_{2}\right)^{s}}{\left(\mathrm{t}_{3} p-\mathrm{t}_{1}\right)^{s}} \mathfrak{U}_{*}\left(\mathrm{t}_{1}, \varphi\right)+\frac{\left(\mathrm{t}_{2} p-\mathrm{t}_{1} p\right)^{s}}{\left(\mathrm{t}_{3} p-\mathrm{t}_{1}\right)^{s}} \mathfrak{U}_{*}\left(\mathrm{t}_{3}, \varphi\right) \\
& =\frac{\left(\mathfrak{t}_{3}^{p}-\mathrm{t}_{2} p\right)^{s}}{\left(\mathfrak{t}_{3} p-\mathrm{t}_{1} p\right)^{s}} \mathfrak{U}^{*}\left(\mathfrak{t}_{1}, \varphi\right)+\frac{\left(\mathrm{t}_{2} p-\mathfrak{t}_{1}\right)^{s}}{\left(\mathrm{t}_{3}{ }^{p}-\mathfrak{t}_{1}\right)^{s}} \mathfrak{U}^{*}\left(\mathfrak{t}_{3}, \varphi\right) .
\end{aligned}
$$

From Equation (16), we have

$$
\begin{aligned}
& \left(\mathrm{t}_{3}{ }^{p}-\mathrm{t}_{1}{ }^{p}\right)^{S} \mathfrak{U}_{*}\left(\mathrm{t}_{2}, \varphi\right) \leq\left(\mathrm{t}_{3}{ }^{p}-\mathrm{t}_{2}{ }^{p}\right)^{S} \mathfrak{U}_{*}\left(\mathrm{t}_{1}, \varphi\right)+\left(\mathrm{t}_{2}{ }^{p}-\mathrm{t}_{1}{ }^{p}\right)^{S} \mathfrak{U}_{*}\left(\mathrm{t}_{3}, \varphi\right), \\
& \left(\mathrm{t}_{3}{ }^{p}-\mathrm{t}_{1}{ }^{p}\right)^{\mathcal{S}} \mathfrak{U}^{*}\left(\mathrm{t}_{2}, \varphi\right) \leq\left(\mathrm{t}_{3}{ }^{p}-\mathrm{t}_{2}{ }^{p}\right)^{\mathcal{S}} \mathfrak{U}^{*}\left(\mathrm{t}_{1}, \varphi\right)+\left(\mathrm{t}_{2}{ }^{p}-\mathrm{t}_{1}{ }^{p}\right)^{\mathcal{S}} \mathfrak{U}^{*}\left(\mathrm{t}_{3}, \varphi\right),
\end{aligned}
$$

that is

$$
\begin{gathered}
{\left[\left(\mathrm{t}_{3}{ }^{p}-\mathrm{t}_{1}{ }^{p}\right)^{s} \mathfrak{U}_{*}\left(\mathrm{t}_{2}, \varphi\right),\left(\mathrm{t}_{3}{ }^{p}-\mathrm{t}_{1}{ }^{p}\right)^{s} \mathfrak{U}^{*}\left(\mathrm{t}_{2}, \varphi\right)\right]} \\
\leq_{I}\left[\left(\mathrm{t}_{3}{ }^{p}-\mathrm{t}_{2}{ }^{p}\right)^{\mathcal{S}} \mathfrak{U}_{*}\left(\mathrm{t}_{1}, \varphi\right)+\left(\mathrm{t}_{2}{ }^{p}-\mathrm{t}_{1}{ }^{p}\right)^{s} \mathfrak{U}_{*}\left(\mathrm{t}_{3}, \varphi\right),\left(\mathrm{t}_{3}{ }^{p}-\mathrm{t}_{2}{ }^{p}\right)^{s} \mathfrak{U}^{*}\left(\mathrm{t}_{1}, \varphi\right)+\left(\mathrm{t}_{2}{ }^{p}-\mathrm{t}_{1}{ }^{p}\right)^{\mathcal{s}} \mathfrak{U}^{*}\left(\mathrm{t}_{3}, \varphi\right)\right] .
\end{gathered}
$$

Hence,

$$
\left(\mathrm{t}_{3}^{p}-\mathrm{t}_{1}^{p}\right)^{s} \mathfrak{U}\left(\mathrm{t}_{2}\right) \preccurlyeq\left(\mathrm{t}_{3}^{p}-\mathrm{t}_{2}^{p}\right)^{s} \mathfrak{U}\left(\mathrm{t}_{1}\right)+\left(\mathrm{t}_{2}^{p}-\mathrm{t}_{1}^{p}\right)^{s} \mathfrak{U}\left(\mathrm{t}_{3}\right)
$$

A refinement of Jensen type inequality for $(p, s)$-convex $F-I-V-F$ is given in the following theorem.

Theorem 5. Let $s \in[0,1], \omega_{j} \in \mathbb{R}^{+}, \mathrm{t}_{j} \in[\mathrm{t}, \mathrm{s}],(j=1,2,3, \ldots, k, k \geq 2)$ and $\mathfrak{U}:[\mathrm{t}, \mathrm{s}] \rightarrow \mathbb{F}_{C}(\mathbb{R})$ be a $(p, s)$-convex $F-I-V-F$, whose $\varphi$-levels define the family of $I-V$ - $F \mathrm{~s}$ $\mathfrak{U}_{\varphi}:[\mathrm{t}, \mathrm{s}] \subset \mathbb{R} \rightarrow \mathcal{K}_{C}{ }^{+}$are given by $\mathfrak{U}_{\varphi}(\varkappa)=\left[\mathfrak{U}_{*}(\varkappa, \varphi), \mathfrak{U}^{*}(\varkappa, \varphi)\right]$ for all $\in[\mathrm{t}$, s] and for all $\varphi \in[0,1]$. If $(L, U) \subseteq[\mathrm{t}, \mathrm{s}]$, then

$$
\sum_{j=1}^{k}\left(\frac{\omega_{j}}{W_{k}}\right)^{s} \mathfrak{U}\left(\mathbf{t}_{j}\right) \preccurlyeq \sum_{j=1}^{k}\left(\left(\frac{U^{p}-\mathbf{t}_{j}^{p}}{U^{p}-L^{p}}\right)^{s}\left(\frac{\omega_{j}}{W_{k}}\right)^{s} \mathfrak{U}(L, \varphi)+\left(\frac{\mathbf{t}_{j}^{p}-L^{p}}{U^{p}-L^{p}}\right)^{s}\left(\frac{\omega_{j}}{W_{k}}\right)^{s} \mathfrak{U}(U, \varphi)\right),
$$

where $W_{k}=\sum_{j=1}^{k} \omega_{j}$. If $\mathfrak{U}$ is $(p, s)$-concave $F-I-V-F$, then inequality Equation (17) is reversed.

Proof. Consider $\mathrm{t}_{j}$ such that $L<\mathrm{t}_{j}<U(j=1,2,3, \ldots, k)$. Then, by hypothesis and inequality Equation (15), we have

$$
\mathfrak{U}\left(\mathbf{t}_{j}\right) \leq\left(\frac{U^{p}-\mathbf{t}_{j}^{p}}{U^{p}-L^{p}}\right)^{s} \mathfrak{U}(L, \varphi)+\left(\frac{\mathbf{t}_{j}^{p}-L^{p}}{U^{p}-L^{p}}\right)^{s} \mathfrak{U}(U, \varphi) .
$$


Therefore, for each $\varphi \in[0,1]$, we have

$$
\begin{aligned}
& \mathfrak{U}_{*}\left(\mathbf{t}_{j}, \varphi\right) \leq\left(\frac{U^{p}-\mathbf{t}_{j}^{p}}{U^{p}-L^{p}}\right)^{S} \mathfrak{U}_{*}(L, \varphi)+\left(\frac{\mathfrak{t}_{j}^{p}-L^{p}}{U^{p}-L^{p}}\right)^{S} \mathfrak{U}_{*}(U, \varphi), \\
& \mathfrak{U}^{*}\left(\mathbf{t}_{j}, \varphi\right) \leq\left(\frac{U^{p}-\mathfrak{t}_{j}^{p}}{U^{p}-L^{p}}\right)^{S} \mathfrak{U}^{*}(L, \varphi)+\left(\frac{\mathfrak{t}_{j} L^{p}}{U^{p}-L^{p}}\right)^{S} \mathfrak{U}^{*}(U, \varphi) .
\end{aligned}
$$

The above inequality can be written as

$$
\begin{aligned}
& \left(\frac{\omega_{j}}{W_{k}}\right)^{s} \mathfrak{U}_{*}\left(\mathbf{t}_{j}, \varphi\right) \leq\left(\frac{U^{p}-\mathbf{t}_{j}^{p}}{U^{p}-L^{p}}\right)^{s}\left(\frac{\omega_{j}}{W_{k}}\right)^{s} \mathfrak{U}_{*}(L, \varphi)+\left(\frac{\mathrm{t}_{j}{ }^{p}-L^{p}}{U^{p}-L^{p}}\right)^{s}\left(\frac{\omega_{j}}{W_{k}}\right)^{s} \mathfrak{U}_{*}(U, \varphi), \\
& \left(\frac{\omega_{j}}{W_{k}}\right)^{S} \mathfrak{U}^{*}\left(\mathbf{t}_{j}, \varphi\right) \leq\left(\frac{U^{p}-t_{j}^{p}}{U^{p}-L^{p}}\right)^{s}\left(\frac{\omega_{j}}{W_{k}}\right)^{S} \mathfrak{U}^{*}(L, \varphi)+\left(\frac{\mathrm{t}_{j}{ }^{p}-L^{p}}{U^{p}-L^{p}}\right)^{S}\left(\frac{\omega_{j}}{W_{k}}\right)^{S} \mathfrak{U}^{*}(U, \varphi)
\end{aligned}
$$

Taking the sum of all inequalities (18) for $j=1,2,3, \ldots, k$, we have

$$
\begin{aligned}
& \sum_{j=1}^{k}\left(\frac{\omega_{j}}{W_{k}}\right)^{S} \mathfrak{U}_{*}\left(\mathfrak{t}_{j}, \varphi\right) \leq \sum_{j=1}^{k}\left(\left(\frac{U^{p}-\mathbf{t}_{j}^{p}}{U^{p}-L^{p}}\right)^{s}\left(\frac{\omega_{j}}{W_{k}}\right)^{S} \mathfrak{U}_{*}(L, \varphi)+\left(\frac{\mathrm{t}_{j}{ }^{p}-L^{p}}{U^{p}-L^{p}}\right)^{s}\left(\frac{\omega_{j}}{W_{k}}\right)^{S} \mathfrak{U}_{*}(U, \varphi)\right), \\
& \sum_{j=1}^{k}\left(\frac{\omega_{j}}{W_{k}}\right)^{s} \mathfrak{U}^{*}\left(\mathfrak{t}_{j}, \varphi\right) \leq \sum_{j=1}^{k}\left(\left(\frac{U^{p}-\mathbf{t}_{j}^{p}}{U^{p}-L^{p}}\right)^{s}\left(\frac{\omega_{j}}{W_{k}}\right)^{s} \mathfrak{U}^{*}(L, \varphi)+\left(\frac{\mathrm{t}_{j}{ }^{p}-L^{p}}{U^{p}-L^{p}}\right)^{s}\left(\frac{\omega_{j}}{W_{k}}\right)^{s} \mathfrak{U}^{*}(U, \varphi)\right),
\end{aligned}
$$

that is

$$
\begin{aligned}
& \sum_{j=1}^{k}\left(\frac{\omega_{j}}{W_{k}}\right)^{S} \mathfrak{U}_{\varphi}\left(\mathrm{t}_{j}\right)=\left[\sum_{j=1}^{k}\left(\frac{\omega_{j}}{W_{k}}\right)^{S} \mathfrak{U}_{*}\left(\mathrm{t}_{j}, \varphi\right), \sum_{j=1}^{k}\left(\frac{\omega_{j}}{W_{k}}\right)^{s} \mathfrak{U}^{*}\left(\mathfrak{t}_{j}, \varphi\right)\right]
\end{aligned}
$$

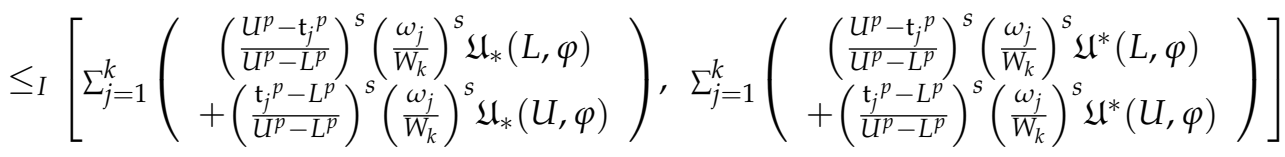

$$
\begin{aligned}
& =\sum_{j=1}^{k}\left(\frac{U^{p}-\mathfrak{t}_{j}^{p}}{U^{p}-L^{p}}\right)^{s}\left(\frac{\omega_{j}}{W_{k}}\right)^{s}\left[\mathfrak{U}_{*}(L, \varphi), \mathfrak{U}^{*}(L, \varphi)\right]+\sum_{j=1}^{k}\left(\frac{\mathfrak{t}_{j}^{p}-L^{p}}{U^{p}-L^{p}}\right)^{s}\left(\frac{\omega_{j}}{W_{k}}\right)^{s}\left[\mathfrak{U}_{*}(U, \varphi), \mathfrak{U}^{*}(U, \varphi)\right] \\
& =\sum_{j=1}^{k}\left(\frac{U^{p}-\mathbf{t}_{j}^{p}}{U^{p}-L^{p}}\right)^{s}\left(\frac{\omega_{j}}{W_{k}}\right)^{s} \mathfrak{U}_{\varphi}(L)+\sum_{j=1}^{k}\left(\frac{\mathbf{t}_{j}^{p}-L^{p}}{U^{p}-L^{p}}\right)^{s}\left(\frac{\omega_{j}}{W_{k}}\right)^{s} \mathfrak{U}_{\varphi}(U) .
\end{aligned}
$$

Thus,

$$
\sum_{j=1}^{k}\left(\frac{\omega_{j}}{W_{k}}\right)^{s} \mathfrak{U}\left(\mathrm{t}_{j}\right) \preccurlyeq \sum_{j=1}^{k}\left(\left(\frac{U^{p}-\mathrm{t}_{j}^{p}}{U^{p}-L^{p}}\right)^{s}\left(\frac{\omega_{j}}{W_{k}}\right)^{s} \mathfrak{U}(L)+\left(\frac{\mathrm{t}_{j}^{p}-L^{p}}{U^{p}-L^{p}}\right)^{s}\left(\frac{\omega_{j}}{W_{k}}\right)^{s} \mathfrak{U}(U)\right),
$$

and this completes the proof.

We now consider some special cases of Theorems 3 and 5 .

If $\mathfrak{U}_{*}(\varkappa, \varphi)=\mathfrak{U}_{*}(\varkappa, \varphi)$, then Theorems 3 and 5 reduce to the following results:

Corollary 2 ([21]). (Jensen inequality for $(p, s)$-convex function) Let $s \in[0,1], \omega_{j} \in \mathbb{R}^{+}$, $\mathrm{t}_{j} \in[\mathrm{t}, \mathrm{s}],(j=1,2,3, \ldots, k, k \geq 2)$ and let $\mathfrak{U}:[\mathrm{t}, \mathrm{s}] \rightarrow \mathbb{R}^{+}$be a non-negative real-valued function. If $\mathfrak{U}$ is a $(p, s)$-convex function, then

$$
\mathfrak{U}\left(\left[\frac{1}{W_{k}} \sum_{j=1}^{k} \omega_{j} \mathbf{t}_{j}\right]^{\frac{1}{p}}\right) \leq \sum_{j=1}^{k}\left(\frac{\omega_{j}}{W_{k}}\right)^{s} \mathfrak{U}\left(\mathbf{t}_{j}\right),
$$

where $W_{k}=\sum_{j=1}^{k} \omega_{j}$. If $\mathfrak{U}$ is $(p, s)$-concave function, then inequality (19) is reversed. 
Corollary 3. Let $s \in[0,1], \omega_{j} \in \mathbb{R}^{+}, \mathrm{t}_{j} \in[\mathrm{t}, \mathrm{s}],(j=1,2,3, \ldots, k, k \geq 2)$, and $\mathfrak{U}:[\mathrm{t}, \mathrm{s}] \rightarrow \mathbb{R}^{+}$ be a non-negative real-valued function. If $\mathfrak{U}$ is a $(p, s)$-convex function and $\mathrm{t}_{1}, \mathrm{t}_{2}, \ldots, \mathrm{t}_{j} \in$ $(L, U) \subseteq[\mathrm{t}, \mathrm{s}]$, then

$$
\sum_{j=1}^{k}\left(\frac{\omega_{j}}{W_{k}}\right)^{s} \mathfrak{U}\left(\mathrm{t}_{j}\right) \leq \sum_{j=1}^{k}\left(\left(\frac{U^{p}-\mathrm{t}_{j}^{p}}{U^{p}-L^{p}}\right)^{s}\left(\frac{\omega_{j}}{W_{k}}\right)^{s} \mathfrak{U}(L)+\left(\frac{\mathrm{t}_{j}{ }^{p}-L^{p}}{U^{p}-L^{p}}\right)^{s}\left(\frac{\omega_{j}}{W_{k}}\right)^{s} \mathfrak{U}(U)\right),
$$

where $W_{k}=\sum_{j=1}^{k} \omega_{j}$. If $\mathfrak{U}$ is a $(p, s)$-concave function, then inequality (20) is reversed.

\section{Hermite-Hadamard Type Inequalities for $(p, s)$-Convex F-I-V-F in the Second Sense}

In this section, we will continue with the $\mathrm{H}-\mathrm{H}$ inequality for $(p, s)$-convex fuzzy-I-V-Fs as well as the fuzzy-interval $\mathrm{H}-\mathrm{H}$ Fejér inequality for $(p, s)$-convex fuzzy-I-V-Fs using the fuzzy order relation. Firstly, we start with the following $\mathrm{H}-\mathrm{H}$ inequality for $(p, s)$-convex fuzzy-I-V-Fs:

Theorem 6. Let $\mathfrak{U}:[\mathrm{t}, \mathrm{s}] \rightarrow \mathbb{F}_{C}(\mathbb{R})$ be a $(p, s)$-convex $F-I-V$-F, whose $\varphi$-levels define the family of $I-V$-Fs. $\mathfrak{U}_{\varphi}:[\mathrm{t}, \mathrm{s}] \subset \mathbb{R} \rightarrow \mathcal{K}_{C^{+}}$are given by $\mathfrak{U}_{\varphi}(\varkappa)=\left[\mathfrak{U}_{*}(\varkappa, \varphi), \mathfrak{U}^{*}(\varkappa, \varphi)\right]$ for all $\in[\mathrm{t}, \mathrm{s}]$ and for all $\varphi \in[0,1]$. If $\mathfrak{U} \in \mathcal{F} \mathcal{R}_{([\mathrm{t}, \mathrm{s}]}$, then

$$
2^{s-1} \mathfrak{U}\left(\left[\frac{\mathrm{t}^{p}+\mathrm{s}^{p}}{2}\right]^{\frac{1}{p}}\right) \preccurlyeq \frac{p}{\mathrm{~s}^{p}-\mathrm{t}^{p}}(F R) \int_{\mathrm{t}}^{\mathrm{s}} \varkappa^{p-1} \mathfrak{U}(\varkappa) d \varkappa \leq_{p} \frac{\mathfrak{U}(\mathrm{t}) \widetilde{+} \mathfrak{U}(\mathrm{s})}{\mathrm{s}+1} .
$$

If $\mathfrak{U}$ is a $(p, s)$-concave $F-I-V-F$, then

$$
2^{s-1} \mathfrak{U}\left(\left[\frac{\mathrm{t}^{p}+\mathrm{s}^{p}}{2}\right]^{\frac{1}{p}}\right) \succcurlyeq \frac{p}{\mathrm{~s}^{p}-\mathrm{t}^{p}}(F R) \int_{\mathrm{t}}^{\mathrm{s}} \varkappa^{p-1} \mathfrak{U}(\varkappa) d \varkappa \succcurlyeq \frac{\mathfrak{U}(\mathrm{t}) \widetilde{+} \mathfrak{U}(\mathrm{s})}{s+1} .
$$

Proof. Let $\mathfrak{U}$ be a $(p, s)$-convex $F-I-V-F$. Then, by hypothesis, we have

$$
2^{s} \mathfrak{U}\left(\left[\frac{\mathrm{t}^{p}+\mathrm{s}^{p}}{2}\right]^{\frac{1}{p}}\right) \preccurlyeq \mathfrak{U}\left(\left[\zeta \mathrm{t}^{p}+(1-\zeta) \mathbf{s}^{p}\right]^{\frac{1}{p}}\right) \widetilde{+} \mathfrak{U}\left(\left[(1-\zeta) \mathrm{t}^{p}+\zeta \mathbf{s}^{p}\right]^{\frac{1}{p}}\right) .
$$

Therefore, for each $\varphi \in[0,1]$, we have

$$
\begin{aligned}
& 2^{s} \mathfrak{U}_{*}\left(\left[\frac{\mathrm{t}^{p}+\mathrm{s}^{p}}{2}\right]^{\frac{1}{p}}, \varphi\right) \leq \mathfrak{U}_{*}\left(\left[\zeta \mathrm{t}^{p}+(1-\zeta) \mathrm{s}^{p}\right]^{\frac{1}{p}}, \varphi\right)+\mathfrak{U}_{*}\left((1-\zeta) \mathrm{t}^{p}+\zeta \mathrm{s}^{p}, \varphi\right), \\
& 2^{s} \mathfrak{U}^{*}\left(\left[\frac{\mathrm{t}^{p}+\mathrm{s}^{p}}{2}\right]^{\frac{1}{p}}, \varphi\right) \leq \mathfrak{U}^{*}\left(\left[\zeta \mathrm{t}^{p}+(1-\zeta) \mathrm{s}^{p}\right]^{\frac{1}{p}}, \varphi\right)+\mathfrak{U}^{*}\left((1-\zeta) \mathrm{t}^{p}+\zeta \mathrm{s}^{p}, \varphi\right) .
\end{aligned}
$$

Then,

$$
\begin{aligned}
& 2^{s} \int_{0}^{1} \mathfrak{U}_{*}\left(\left[\frac{\mathrm{t}^{p}+\mathrm{s}^{p}}{2}\right]^{\frac{1}{p}}, \varphi\right) d \zeta \leq \int_{0}^{1} \mathfrak{U}_{*}\left(\left[\zeta \mathrm{t}^{p}+(1-\zeta) \mathbf{s}^{p}\right]^{\frac{1}{p}}, \varphi\right) d \zeta+\int_{0}^{1} \mathfrak{U}_{*}\left((1-\zeta) \mathbf{t}^{p}+\zeta \mathbf{s}^{p}, \varphi\right) d \zeta, \\
& 2^{s} \int_{0}^{1} \mathfrak{U}^{*}\left(\left[\frac{\left[\mathrm{t}^{p}+\mathrm{s}^{p}\right.}{2}\right]^{\frac{1}{p}}, \varphi\right) d \zeta \leq \int_{0}^{1} \mathfrak{U}^{*}\left(\left[\zeta \mathrm{t}^{p}+(1-\zeta) \mathbf{s}^{p}\right]^{\frac{1}{p}}, \varphi\right) d \zeta+\int_{0}^{1} \mathfrak{U}^{*}\left((1-\zeta) \mathbf{t}^{p}+\zeta \mathbf{s}^{p}, \varphi\right) d \zeta .
\end{aligned}
$$

It follows that

$$
\begin{aligned}
& 2^{s-1} \mathfrak{U}_{*}\left(\left[\frac{\mathrm{t}^{p}+\mathrm{s}^{p}}{2}\right]^{\frac{1}{p}}, \varphi\right) \leq \frac{p}{s^{p}-\mathrm{t}^{p}} \int_{\mathrm{t}}^{\mathrm{s}} \varkappa^{p-1} \mathfrak{U}_{*}(\varkappa, \varphi) d \varkappa, \\
& 2^{s-1} \mathfrak{U}^{*}\left(\left[\frac{\mathrm{t}^{p}+\mathrm{s}^{p}}{2}\right]^{\frac{1}{p}}, \varphi\right) \leq \frac{p}{\mathrm{~s}^{p}-\mathrm{t}^{p}} \int_{\mathrm{t}}^{\mathrm{s}} \varkappa^{p-1} \mathfrak{U}^{*}(\varkappa, \varphi) d \varkappa .
\end{aligned}
$$

That is, 


$$
2^{s-1}\left[\mathfrak{U}_{*}\left(\left[\frac{\mathrm{t}^{p}+\mathrm{s}^{p}}{2}\right]^{\frac{1}{p}}, \varphi\right), \mathfrak{U}^{*}\left(\left[\frac{\mathrm{t}^{p}+\mathrm{s}^{p}}{2}\right]^{\frac{1}{p}}, \varphi\right)\right] \leq_{I} \frac{p}{\mathrm{~s}^{p}-\mathrm{t}^{p}}\left[\int_{\mathrm{t}}^{\mathrm{s}} \varkappa^{p-1} \mathfrak{U}_{*}(\varkappa, \varphi) d \varkappa, \int_{\mathrm{t}}^{\mathrm{s}} \varkappa^{p-1} \mathfrak{U}^{*}(\varkappa, \varphi) d \varkappa\right] .
$$

Thus,

$$
2^{s-1} \mathfrak{U}\left(\left[\frac{\mathrm{t}^{p}+\mathrm{s}^{p}}{2}\right]^{\frac{1}{p}}\right) \preccurlyeq \frac{p}{\mathrm{~s}^{p}-\mathrm{t}^{p}}(F R) \int_{\mathrm{t}}^{\mathrm{s}} \varkappa^{p-1} \mathfrak{U}(\varkappa) d \varkappa .
$$

In a similar way as above, we have

$$
\frac{p}{\mathrm{~s}^{p}-\mathrm{t}^{p}}(F R) \int_{\mathrm{t}}^{\mathrm{s}} \varkappa^{p-1} \mathfrak{U}(\varkappa) d \varkappa \preccurlyeq \frac{1}{s+1}[\mathfrak{U}(\mathrm{t}) \widetilde{+} \mathfrak{U}(\mathrm{s})] .
$$

Combining Equations (23) and (24), we have

$$
2^{s-1} \mathfrak{U}\left(\left[\frac{\mathrm{t}^{p}+\mathrm{s}^{p}}{2}\right]^{\frac{1}{p}}\right) \preccurlyeq \frac{p}{\mathrm{~s}^{p}-\mathrm{t}^{p}}(F R) \int_{\mathrm{t}}^{\mathrm{s}} \varkappa^{p-1} \mathfrak{U}(\varkappa) d \varkappa \preccurlyeq \frac{1}{s+1}[\mathfrak{U}(\mathrm{t}) \widetilde{+} \mathfrak{U}(\mathrm{s})] .
$$

Hence, we obtain the required result.

Remark 4. On the basis of Theorem 6, we consider the certain the special situation as below:

- If we attempt to take $\mathfrak{U}_{*}(\varkappa, \varphi)=\mathfrak{U}^{*}(\varkappa, \varphi)$ with $\varphi=1$, then we achieve the $(p, s)$ convex function, see [9];

- If we attempt to take $s=1$, then we achieve the result for $p$-convex F-I-V-F-:

$$
\mathfrak{U}\left(\left[\frac{\mathrm{t}^{p}+\mathrm{s}^{p}}{2}\right]^{\frac{1}{p}}\right) \preccurlyeq \frac{p}{\mathrm{~s}^{p}-\mathrm{t}^{p}}(F R) \int_{\mathrm{t}}^{\mathrm{s}} \varkappa^{p-1} \mathfrak{U}(\varkappa) d \varkappa \preccurlyeq \frac{\mathfrak{U}(\mathrm{t}) \widetilde{+} \mathfrak{U}(\mathrm{s})}{2} ;
$$

- If we attempt to take $p=1$, then we achieve the result for s-convex $F-I-V-F$, see [13]:

$$
\mathfrak{U}\left(\frac{\mathrm{t}+\mathrm{s}}{2}\right) \preccurlyeq \frac{1}{\mathrm{~s}-\mathrm{t}}(F R) \int_{\mathrm{t}}^{\mathrm{s}} \mathfrak{U}(\varkappa) d \varkappa \preccurlyeq \frac{\mathfrak{U}(\mathrm{t}) \widetilde{+} \mathfrak{U}(\mathrm{s})}{\mathrm{s}+1} ;
$$

- If we attempt to take $s=1$ and $p=1$, then we achieve the result for $p$-convex F-I-V-F, see [13]:

$$
\mathfrak{U}\left(\frac{\mathrm{t}+\mathrm{s}}{2}\right) \preccurlyeq \frac{1}{\mathrm{~s}-\mathrm{t}}(F R) \int_{\mathrm{t}}^{\mathrm{s}} \mathfrak{U}(\varkappa) d \varkappa \preccurlyeq \frac{\mathfrak{U}(\mathrm{t}) \widetilde{+} \mathfrak{U}(\mathrm{s})}{2} ;
$$

- If we attempt to take $\mathfrak{U}_{*}(\varkappa, \varphi)=\mathfrak{U}^{*}(\varkappa, \varphi)$ with $\varphi=1$, then we acquire the result for classical $(p, s)$-convex function, see [21]:

$$
2^{s-1} \mathfrak{U}\left(\left[\frac{\mathrm{t}^{p}+\mathrm{s}^{p}}{2}\right]^{\frac{1}{p}}\right) \leq \frac{p}{\mathrm{~s}^{p}-\mathrm{t}^{p}}(R) \int_{\mathrm{t}}^{\mathrm{s}} \varkappa^{p-1} \mathfrak{U}(\varkappa) d \varkappa \leq \frac{1}{s+1}[\mathfrak{U}(\mathrm{t}) \widetilde{+} \mathfrak{U}(\mathrm{s})]
$$

- If we attempt to take $\mathfrak{U}_{*}(\varkappa, \varphi)=\mathfrak{U}^{*}(\varkappa, \varphi)$ with $\varphi=1$ and $s=1$, then we acquire the result for classical $p$-convex function:

$$
\mathfrak{U}\left(\left[\frac{\mathrm{t}^{p}+\mathrm{s}^{p}}{2}\right]^{\frac{1}{p}}\right) \leq \frac{p}{\mathrm{~s}^{p}-\mathrm{t}^{p}}(R) \int_{\mathrm{t}}^{\mathrm{s}} \varkappa^{p-1} \mathfrak{U}(\varkappa) d \varkappa \leq \frac{\mathfrak{U}(\mathrm{t})+\mathfrak{U}(\mathrm{s})}{2} ;
$$


- If we attempt to take $\mathfrak{U}_{*}(\varkappa, \varphi)=\mathfrak{U}^{*}(\varkappa, \varphi)$ with, $\varphi=1, p=1$ and $s=1$, then we acquire the result for classical convex function:

$$
\mathfrak{U}\left(\frac{\mathrm{t}+\mathrm{s}}{2}\right) \leq \frac{1}{\mathrm{~s}-\mathrm{t}}(R) \int_{\mathrm{t}}^{\mathrm{s}} \mathfrak{U}(\varkappa) d \varkappa \leq \frac{\mathfrak{U}(\mathrm{t})+\mathfrak{U}(\mathrm{s})}{2} .
$$

Example 2. Let $p$ be an odd number and $s \in[0,1]$, and the $F-I-V-F \mathfrak{U}:[\mathrm{t}, \mathrm{s}]=[2,3] \rightarrow \mathbb{F}_{C}(\mathbb{R})$ defined by

$$
\mathfrak{U}(\varkappa)(\sigma)=\left\{\begin{array}{cc}
\frac{\sigma}{\left(2-\varkappa^{\frac{p}{2}}\right)}, \quad \sigma \in\left[0,2-\varkappa^{\frac{p}{2}}\right] \\
\frac{2\left(2-\varkappa^{\frac{p}{2}}\right)-\sigma}{\left(2-\varkappa^{\frac{p}{2}}\right)}, \quad \sigma \in\left(2-\varkappa^{\frac{p}{2}}, 2\left(2-\varkappa^{\frac{p}{2}}\right)\right] \\
0, \quad \text { otherwise. }
\end{array}\right.
$$

Then, for each $\varphi \in[0,1]$, we have $\mathfrak{U}_{\varphi}(\varkappa)=\left[\varphi\left(2-\varkappa^{\frac{p}{2}}\right),(2-\varphi)\left(2-\varkappa^{\frac{p}{2}}\right)\right]$. Since end point functions $\mathfrak{U}_{*}(\varkappa, \varphi)=\varphi\left(2-\varkappa^{\frac{p}{2}}\right), \mathfrak{U}^{*}(\varkappa, \varphi)=(2-\varphi)\left(2-\varkappa^{\frac{p}{2}}\right)$ are $(p, s)$ convex functions for each $\varphi \in[0,1]$. Then, $\mathfrak{U}(\varkappa)$ is $(p, s)$-convex $F-I-V-F$. We now compute the following:

$$
\begin{gathered}
2^{s-1} \mathfrak{U}_{*}\left(\left[\frac{\mathrm{t}^{p}+\mathrm{s}^{p}}{2}\right]^{\frac{1}{p}}, \varphi\right)=\frac{4-\sqrt{10}}{2} \varphi, \\
2^{s-1} \mathfrak{U}^{*}\left(\left[\frac{\mathrm{t}^{p}+\mathrm{s}^{p}}{2}\right]^{\frac{1}{p}}, \varphi\right)=\frac{4-\sqrt{10}}{2}(2-\varphi), \\
\frac{p}{\mathrm{~s}^{p}-\mathrm{t}^{p}} \int_{\mathrm{t}}^{s} \varkappa^{p-1} \mathfrak{U}_{*}(\varkappa, \varphi) d \varkappa=\varphi \int_{2}^{3}\left(2-\varkappa^{\frac{p}{2}}\right) d \varkappa=\frac{21}{50} \varphi, \\
\frac{p}{\mathrm{~s}^{p}-\mathrm{t}^{p}} \int_{\mathrm{t}}^{\mathcal{s}} \varkappa^{p-1} \mathfrak{U}^{*}(\varkappa, \varphi) d \varkappa=(2-\varphi) \int_{2}^{3}\left(2-\varkappa^{\frac{p}{2}}\right) d \varkappa=\frac{21}{50}(2-\varphi), \\
\frac{\mathfrak{U}_{*}(\mathrm{t}, \varphi)+\mathfrak{U}_{*}(\mathrm{~s}, \varphi)}{s+1}=\frac{4-\sqrt{2}-\sqrt{3}}{2} \varphi, \\
\frac{\mathfrak{U}^{*}(\mathrm{t}, \varphi)+\mathfrak{U}^{*}(\mathrm{~s}, \varphi)}{s+1}=\frac{4-\sqrt{2}-\sqrt{3}}{2}(2-\varphi),
\end{gathered}
$$

for all $\varphi \in[0,1]$. That means

$\left[\frac{4-\sqrt{10}}{2} \varphi, \frac{4-\sqrt{10}}{2}(2-\varphi)\right] \leq_{I}\left[\frac{21}{50} \varphi, \frac{21}{50}(2-\varphi)\right] \leq_{I}\left[\frac{4-\sqrt{2}-\sqrt{3}}{2} \varphi, \frac{4-\sqrt{2}-\sqrt{3}}{2}(2-\varphi)\right]$, for all $\varphi \in[0,1]$, and the Theorem 6 has been verified.

Theorem 7. Let $\mathfrak{U}:[\mathrm{t}, \mathrm{s}] \rightarrow \mathbb{F}_{C}(\mathbb{R})$ be a $(p, s)$-convex $F-I-V$ - $F$, whose $\varphi$-levels define the family of $I-V$-Fs $\mathfrak{U}_{\varphi}:[\mathrm{t}, \mathrm{s}] \subset \mathbb{R} \rightarrow \mathcal{K}_{C}+$ are given by $\mathfrak{U}_{\varphi}(\varkappa)=\left[\mathfrak{U}_{*}(\varkappa, \varphi), \mathfrak{U}^{*}(\varkappa, \varphi)\right]$ for all $\in[\mathrm{t}, \mathrm{s}]$ and for all $\varphi \in[0,1]$. If $\mathfrak{U} \in \mathcal{F} \mathcal{R}_{([\mathrm{t}, \mathrm{s}])}$, then

$$
4^{s-1} \mathfrak{U}\left(\left[\frac{\mathrm{t}^{p}+\mathrm{s}^{p}}{2}\right]^{\frac{1}{p}}\right) \preccurlyeq \diamond_{2} \preccurlyeq \frac{p}{\mathrm{~s}^{p}-\mathrm{t}^{p}}(F R) \int_{\mathrm{t}}^{\mathrm{s}} \varkappa^{p-1} \mathfrak{U}(\varkappa) d \varkappa \preccurlyeq \diamond_{1} \preccurlyeq \frac{\mathfrak{U}(\mathrm{t}) \widetilde{+} \mathfrak{U}(\mathrm{s})}{\mathrm{s}+1}\left[\frac{1}{2}+\frac{1}{2^{s}}\right],
$$

where

$$
\diamond_{1}=\frac{\frac{\mathfrak{U}(\mathrm{t}) \widetilde{+} \mathfrak{U}(\mathrm{s})}{2} \widetilde{+} \mathfrak{U}\left(\left[\frac{\mathrm{t}^{p}+\mathrm{s}^{p}}{2}\right]^{\frac{1}{p}}\right)}{s+1}, \diamond_{2}=2^{s-2}\left[\mathfrak{U}\left(\left[\frac{3 \mathrm{t}^{p}+\mathrm{s}^{p}}{4}\right]^{\frac{1}{p}}\right) \widetilde{+} \mathfrak{U}\left(\left[\frac{\mathrm{t}^{p}+3 \mathrm{~s}^{p}}{4}\right]^{\frac{1}{p}}\right)\right],
$$

and $\diamond_{1}=\left[\diamond_{1 *}, \diamond_{1}^{*}\right], \diamond_{2}=\left[\diamond_{2 *}, \diamond_{2}^{*}\right]$. 
Proof. Take $\left[\mathrm{t}^{p}, \frac{\mathrm{t}^{p}+\mathrm{s}^{p}}{2}\right]$, and we have

$$
\begin{gathered}
2^{s} \mathfrak{U}\left(\left[\frac{\zeta t^{p}+(1-\zeta) \frac{\mathfrak{t}^{p}+s^{p}}{2}}{2}+\frac{(1-\zeta) t^{p}+\zeta^{\frac{t^{p}}{2} \mathrm{~s}^{p}}}{2}\right]^{\frac{1}{p}}\right) \\
\preccurlyeq \mathfrak{U}\left(\left[\zeta t^{p}+(1-\zeta) \frac{\mathfrak{t}^{p}+\mathrm{s}^{p}}{2}\right]^{\frac{1}{p}}\right) \widetilde{+} \mathfrak{U}\left(\left[(1-\zeta) \mathfrak{t}^{p}+\zeta \frac{\mathrm{t}^{p}+\mathrm{s}^{p}}{2}\right]^{\frac{1}{p}}\right) .
\end{gathered}
$$

Therefore, for each $\varphi \in[0,1]$, we have

$$
\begin{aligned}
& 2^{s} \mathfrak{U}_{*}\left(\left[\frac{\zeta t^{p}+(1-\zeta) \frac{t^{p}+\mathrm{s}^{p}}{2}}{2}+\frac{(1-\zeta) t^{p}+\zeta^{\frac{t^{p}}{2} \mathrm{~s}^{p}}}{2}\right]^{\frac{1}{p}}, \varphi\right) \\
& \leq \mathfrak{U}_{*}\left(\left[\zeta \mathrm{t}^{p}+(1-\zeta) \frac{\mathrm{t}^{p}+\mathrm{s}^{p}}{2}\right]^{\frac{1}{p}}, \varphi\right)+\mathfrak{U}_{*}\left(\left[(1-\zeta) \mathrm{t}^{p}+\zeta^{\frac{\mathrm{t}^{p}+\mathrm{s}^{p}}{2}}\right]^{\frac{1}{p}}, \varphi\right), \\
& 2^{s} \mathfrak{U}^{*}\left(\left[\frac{\zeta \mathrm{t}^{p}+(1-\zeta) \frac{\mathrm{t}^{p}+\mathrm{s}^{p}}{2}}{2}+\frac{(1-\zeta) \mathrm{t}^{p}+\zeta^{\frac{t^{p}}{2} \mathrm{~s}^{p}}}{2}\right]^{\frac{1}{p}}, \varphi\right) \\
& \leq \mathfrak{U}^{*}\left(\left[\zeta \mathrm{t}^{p}+(1-\zeta) \frac{\mathrm{t}^{p}+\mathrm{s}^{p}}{2}\right]^{\frac{1}{p}}, \varphi\right)+\mathfrak{U}^{*}\left(\left[(1-\zeta) \mathfrak{t}^{p}+\zeta^{\frac{\mathrm{t}^{p}+\mathrm{s}^{p}}{2}}\right]^{\frac{1}{p}}, \varphi\right) .
\end{aligned}
$$

Consequently, we obtain

$$
\begin{aligned}
& 2^{s-2} \mathfrak{U}_{*}\left(\left[\frac{3 \mathrm{t}^{p}+\mathrm{s}^{p}}{4}\right]^{\frac{1}{p}}, \varphi\right) \leq \frac{p}{\mathrm{~s}^{p}-\mathrm{t}^{p}} \int_{\mathrm{t}}^{\frac{\mathrm{t}^{p}+\mathrm{s}^{p}}{2}} \varkappa^{p-1} \mathfrak{U}_{*}(\varkappa, \varphi) d \varkappa, \\
& 2^{s-2} \mathfrak{U}^{*}\left(\left[\frac{3 \mathrm{t}^{p}+\mathrm{s}^{p}}{4}\right]^{\frac{1}{p}}, \varphi\right) \leq \frac{p}{\mathrm{~s}^{p}-\mathrm{t}^{p}} \int_{\mathrm{t}}^{\frac{t^{p}+\mathrm{s}^{p}}{2}} \varkappa^{p-1} \mathfrak{U}^{*}(\varkappa, \varphi) d \varkappa .
\end{aligned}
$$

That is,

$$
\begin{gathered}
2^{s-2}\left[\mathfrak{U}_{*}\left(\left[\frac{3 \mathrm{t}^{p}+\mathrm{s}^{p}}{4}\right]^{\frac{1}{p}}, \varphi\right), \mathfrak{U}^{*}\left(\left[\frac{3 \mathrm{t}^{p}+\mathrm{s}^{p}}{4}\right]^{\frac{1}{p}}, \varphi\right)\right] \\
\leq_{I} \frac{p}{s^{p}-\mathrm{t}^{p}}\left[\int_{\mathrm{t}}^{\frac{t p^{p}+s^{p}}{2}} \varkappa^{p-1} \mathfrak{U}_{*}(\varkappa, \varphi) d \varkappa, \int_{\mathrm{t}}^{\frac{\mathrm{t}^{p}+s^{p}}{2}} \varkappa^{p-1} \mathfrak{U}^{*}(\varkappa, \varphi) d \varkappa\right] .
\end{gathered}
$$

It follows that

$$
2^{s-2} \mathfrak{U}\left(\left[\frac{3 \mathrm{t}^{p}+\mathrm{s}^{p}}{4}\right]^{\frac{1}{p}}\right) \preccurlyeq \frac{p}{\mathrm{~s}^{p}-\mathrm{t}^{p}} \int_{\mathrm{t}}^{\frac{\mathrm{t}^{p}+\mathrm{s}^{p}}{2}} \varkappa^{p-1} \mathfrak{U}(\varkappa) d \varkappa .
$$

In a similar way as above, we have

$$
2^{s-2} \mathfrak{U}\left(\left[\frac{\mathrm{t}^{p}+3 \mathrm{~s}^{p}}{4}\right]^{\frac{1}{p}}\right) \preccurlyeq \frac{p}{\mathrm{~s}^{p}-\mathrm{t}^{p}} \int_{\frac{\mathrm{t}^{p}+\mathrm{s}^{p}}{2}}^{\mathrm{s}} \varkappa^{p-1} \mathfrak{U}(\varkappa) d \varkappa .
$$

Combining Equations (33) and (34), we have

$$
2^{s-2}\left[\mathfrak{U}\left(\left[\frac{3 \mathrm{t}^{p}+\mathrm{s}^{p}}{4}\right]^{\frac{1}{p}}\right) \widetilde{+} \mathfrak{U}\left(\left[\frac{\mathrm{t}^{p}+3 \mathrm{~s}^{p}}{4}\right]^{\frac{1}{p}}\right)\right] \preccurlyeq \frac{p}{\mathrm{~s}^{p}-\mathrm{t}^{p}} \int_{\mathrm{t}}^{\mathrm{s}} \varkappa^{p-1} \mathfrak{U}(\varkappa) d \varkappa .
$$

By using Theorem 6, we have

$$
4^{s-1} \mathfrak{U}\left(\left[\frac{\mathrm{t}^{p}+\mathrm{s}^{p}}{2}\right]^{\frac{1}{p}}\right)=4^{s-1} \mathfrak{U}\left(\left[\frac{1}{2} \cdot \frac{3 \mathrm{t}^{p}+\mathrm{s}^{p}}{4}+\frac{1}{2} \cdot \frac{\mathrm{t}^{p}+3 \mathrm{~s}^{p}}{4}\right]^{\frac{1}{p}}\right) .
$$


Therefore, for each $\varphi \in[0,1]$, we have

$$
\begin{aligned}
& 4^{s-1} \mathfrak{U}_{*}\left(\left[\frac{t^{p}+\mathrm{s}^{p}}{2}\right]^{\frac{1}{p}}, \varphi\right)=4^{s-1} \mathfrak{U}_{*}\left(\left[\frac{1}{2} \cdot \frac{3 \mathrm{t}^{p}+\mathrm{s}^{p}}{4}+\frac{1}{2} \cdot \frac{t^{p}+3 \mathrm{~s}^{p}}{4}\right]^{\frac{1}{p}}, \varphi\right), \\
& 4^{s-1} \mathfrak{U}^{*}\left(\left[\frac{\mathrm{t}^{p}+\mathrm{s}^{p}}{2}\right]^{\frac{1}{p}}, \varphi\right)=4^{s-1} \mathfrak{U}^{*}\left(\left[\frac{1}{2} \cdot \frac{3 \mathrm{t}^{p}+\mathrm{s}^{p}}{4}+\frac{1}{2} \cdot \frac{\mathrm{t}^{p}+3 \mathrm{~s}^{p}}{4}\right]^{\frac{1}{p}}, \varphi\right) \\
& \leq 2^{s-2}\left[\mathfrak{U}_{*}\left(\left[\frac{3 \mathrm{t}^{p}+\mathrm{s}^{p}}{4}\right]^{\frac{1}{p}}, \varphi\right)+\mathfrak{U}_{*}\left(\left[\frac{\mathrm{t}^{p}+3 \mathrm{~s}^{p}}{4}\right]^{\frac{1}{p}}, \varphi\right)\right] \\
& \leq 2^{s-2}\left[\mathfrak{U}^{*}\left(\left[\frac{3 \mathrm{t}^{p}+\mathrm{s}^{p}}{4}\right]^{\frac{1}{p}}, \varphi\right)+\mathfrak{U}^{*}\left(\left[\frac{\mathrm{t}^{p}+3 \mathrm{~s}^{p}}{4}\right]^{\frac{1}{p}}, \varphi\right)\right] \\
& =\diamond_{2 *} \\
& =\triangle_{2}{ }^{*} \\
& \leq \frac{p}{\mathrm{~s}^{p}-\mathrm{t}^{p}} \int_{\mathrm{t}}^{\mathrm{s}} \varkappa^{p-1} \mathfrak{U}_{*}(\varkappa, \varphi) d \varkappa \\
& \leq \frac{p}{s^{p}-t^{p}} \int_{t}^{s} \varkappa^{p-1} \mathfrak{U}^{*}(\varkappa, \varphi) d \varkappa \\
& \leq \frac{1}{s+1}\left[\frac{\mathfrak{U}_{*}(\mathrm{t}, \varphi)+\mathfrak{U}_{*}(\mathrm{~s}, \varphi)}{2}+\mathfrak{U}_{*}\left(\left[\frac{\mathrm{t}^{p}+\mathrm{s}^{p}}{2}\right]^{\frac{1}{p}}, \varphi\right)\right] \\
& \leq \frac{1}{s+1}\left[\frac{\mathfrak{U}^{*}(\mathrm{t}, \varphi)+\mathfrak{U}^{*}(s, \varphi)}{2}+\mathfrak{U}^{*}\left(\left[\frac{\mathrm{t}^{p}+\mathrm{s}^{p}}{2}\right]^{\frac{1}{p}}, \varphi\right)\right] \\
& =\triangleright_{1 *} \\
& =\diamond_{1}{ }^{*} \\
& \leq \frac{1}{s+1}\left[\frac{\mathfrak{U}_{*}(\mathrm{t}, \varphi)+\mathfrak{U}_{*}(\mathrm{~s}, \varphi)}{2}+\frac{1}{2^{\mathrm{s}}}\left(\mathfrak{U}_{*}(\mathrm{t}, \varphi)+\mathfrak{U}_{*}(\mathrm{~s}, \varphi)\right)\right] \\
& \leq \frac{1}{s+1}\left[\frac{\mathfrak{U}^{*}(\mathrm{t}, \varphi)+\mathfrak{U}^{*}(s, \varphi)}{2}+\frac{1}{2^{s}}\left(\mathfrak{U}^{*}(\mathrm{t}, \varphi)+\mathfrak{U}^{*}(\mathrm{~s}, \varphi)\right)\right] \\
& =\frac{1}{s+1}\left[\mathfrak{U}_{*}(\mathrm{t}, \varphi)+\mathfrak{U}_{*}(\mathrm{~s}, \varphi)\right]\left[\frac{1}{2}+\frac{1}{2^{s}}\right] \\
& =\frac{1}{s+1}\left[\mathfrak{U}^{*}(\mathrm{t}, \varphi)+\mathfrak{U}^{*}(\mathrm{~s}, \varphi)\right]\left[\frac{1}{2}+\frac{1}{2^{s}}\right] \text {, }
\end{aligned}
$$

that is

$4^{s-1} \mathfrak{U}\left(\left[\frac{\mathrm{t}^{p}+\mathrm{s}^{p}}{2}\right]^{\frac{1}{p}}\right) \preccurlyeq \diamond_{2} \preccurlyeq \frac{p}{\mathrm{~s}^{p}-\mathrm{t}^{p}}(F R) \int_{\mathrm{t}}^{\mathrm{s}} \varkappa^{p-1} \mathfrak{U}(\varkappa) d \varkappa \preccurlyeq \triangleright_{1} \preccurlyeq \frac{\mathfrak{U}(\mathrm{t}) \widetilde{+} \mathfrak{U}(\mathrm{s})}{s+1}\left[\frac{1}{2}+\frac{1}{2^{s}}\right]$,

hence, the result follows.

Example 3. Let $p$ be an odd number and the $F-I-V-F \mathfrak{U}:[\mathrm{t}, \mathrm{s}]=[2,3] \rightarrow \mathbb{F}_{C}(\mathbb{R})$ defined by, $\mathfrak{U}_{\varphi}(\varkappa)=\left[\varphi\left(2-\varkappa^{\frac{p}{2}}\right),(2-\varphi)\left(2-\varkappa^{\frac{p}{2}}\right)\right]$, as in Example 2, then $\mathfrak{U}(\varkappa)$ is $(p, s)$-convex F-I-V-F and satisfies Equation (21). We have

$$
\mathfrak{U}_{*}(\varkappa, \varphi)=\varphi\left(2-\varkappa^{\frac{p}{2}}\right) \text { and } \mathfrak{U}^{*}(\varkappa, \varphi)=(2-\varphi)\left(2-\varkappa^{\frac{p}{2}}\right)
$$


We now compute the following:

$$
\begin{aligned}
& \frac{\mathfrak{U}_{*}(\mathrm{t}, \varphi)+\mathfrak{U}_{*}(\mathrm{~s}, \varphi)}{s+1}\left[\frac{1}{2}+\frac{1}{2^{\mathrm{s}}}\right]=\frac{4-\sqrt{2}-\sqrt{3}}{2} \varphi, \\
& \frac{\mathfrak{U}^{*}(\mathrm{t}, \varphi)+\mathfrak{U}^{*}(\mathrm{~s}, \varphi)}{s+1}\left[\frac{1}{2}+\frac{1}{2^{\mathrm{s}}}\right]=\frac{4-\sqrt{2}-\sqrt{3}}{2}(2-\varphi), \\
& \diamond_{1 *}=\frac{\frac{\mathfrak{U}_{*}(\mathrm{t}, \varphi)+\mathfrak{U}_{*}(\mathrm{~s}, \varphi)}{2}+\mathfrak{U}_{*}\left(\left[\frac{\mathrm{t}^{p}+\mathrm{s}^{p}}{2}\right]^{\frac{1}{p}}, \varphi\right)}{s+1}=\frac{8-\sqrt{2}-\sqrt{3}-\sqrt{10}}{4} \varphi, \\
& \diamond_{1}{ }^{*}=\frac{\frac{\mathfrak{U}^{*}(\mathrm{t}, \varphi)+\mathfrak{U}^{*}(\mathrm{~s}, \varphi)}{2}+\mathfrak{U}^{*}\left(\left[\frac{\mathrm{t}^{p}+\mathrm{s}^{p}}{2}\right]^{\frac{1}{p}}, \varphi\right)}{s+1}=\frac{8-\sqrt{2}-\sqrt{3}-\sqrt{10}}{4}(2-\varphi), \\
& \diamond_{2 *}=2^{s-2}\left[\mathfrak{U}_{*}\left(\left[\frac{3 \mathrm{t}^{p}+\mathrm{s}^{p}}{4}\right]^{\frac{1}{p}}, \varphi\right)+\mathfrak{U}_{*}\left(\left[\frac{\mathrm{t}^{p}+3 \mathrm{~s}^{p}}{4}\right]^{\frac{1}{p}}, \varphi\right)\right]=\frac{5-\sqrt{11}}{4} \varphi, \\
& \diamond_{2}{ }^{*}=2^{s-2}\left[\mathfrak{U}^{*}\left(\left[\frac{3 \mathrm{t}^{p}+\mathrm{s}^{p}}{4}\right]^{\frac{1}{p}}, \varphi\right)+\mathfrak{U}^{*}\left(\left[\frac{\mathrm{t}^{p}+3 \mathrm{~s}^{p}}{4}\right]^{\frac{1}{p}}, \varphi\right)\right]=\frac{5-\sqrt{11}}{4}(2-\varphi), \\
& 4^{s-1} \mathfrak{U}_{*}\left(\left[\frac{\mathrm{t}^{p}+\mathrm{s}^{p}}{2}\right]^{\frac{1}{p}}, \varphi\right)=\frac{4-\sqrt{10}}{2} \varphi, \\
& 4^{s-1} \mathfrak{U}^{*}\left(\left[\frac{\mathrm{t}^{p}+\mathrm{s}^{p}}{2}\right]^{\frac{1}{p}}, \varphi\right)=\frac{4-\sqrt{10}}{2}(2-\varphi) .
\end{aligned}
$$

Then, we obtain that

$$
\begin{gathered}
\frac{4-\sqrt{10}}{2} \varphi \leq \frac{5-\sqrt{11}}{4} \varphi \leq \frac{21}{50} \varphi \leq \frac{8-\sqrt{2}-\sqrt{3}-\sqrt{10}}{4} \varphi \leq \frac{4-\sqrt{2}-\sqrt{3}}{2} \varphi, \\
\frac{4-\sqrt{10}}{2}(2-\varphi) \leq \frac{5-\sqrt{11}}{4}(2-\varphi) \leq \frac{21}{50}(2-\varphi) \leq \frac{8-\sqrt{2}-\sqrt{3}-\sqrt{10}}{4}(2-\varphi) \leq \frac{4-\sqrt{2}-\sqrt{3}}{2}(2-\varphi) .
\end{gathered}
$$

Hence, Theorem 7 is verified.

The next Theorems 8 and 9 give the second $\mathrm{H}-\mathrm{H}$ Fejér inequality and the first $\mathrm{H}-\mathrm{H}$ Fejér inequality for $(p, s)$-convex $F-I-V-F$, respectively.

Theorem 8. (Second H-H Fejér inequality for $(p, s)$-convex $F-I-V-F)$ Let $\mathfrak{U}:[\mathrm{t}, \mathrm{s}] \rightarrow \mathbb{F}_{C}(\mathbb{R})$ be a $(p, s)$-convex $F-I-V-F$ with $\mathrm{t}<\mathrm{s}$, whose $\varphi$-levels define the family of $I-V$-Fs $\mathfrak{U}_{\varphi}:[\mathrm{t}, \mathrm{s}] \subset \mathbb{R} \rightarrow \mathcal{K}_{C}{ }^{+}$are given by $\mathfrak{U}_{\varphi}(\varkappa)=\left[\mathfrak{U}_{*}(\varkappa, \varphi), \mathfrak{U}^{*}(\varkappa, \varphi)\right]$ for all $\varkappa \in[\mathrm{t}$, s] and for all $\varphi \in[0,1]$. If $\mathfrak{U} \in \mathcal{F} \mathcal{R}_{([\mathrm{t}, \mathrm{s}])}$ and $\Psi:[\mathrm{t}, \mathrm{s}] \rightarrow \mathbb{R}, \Psi(\varkappa) \geq 0, p$-symmetric with respect to $\left[\frac{\mathrm{t}^{p}+\mathrm{s}^{p}}{2}\right]^{\frac{1}{p}}$, then

$$
\frac{p}{\mathrm{~s}^{p}-\mathrm{t}^{p}}(F R) \int_{\mathrm{t}}^{\mathrm{s}} \varkappa^{p-1} \mathfrak{U}(\varkappa) \Psi(\varkappa) d \preccurlyeq[\mathfrak{U}(\mathrm{t}) \widetilde{+} \mathfrak{U}(\mathrm{s})] \int_{0}^{1} \zeta^{s} \Psi\left(\left[(1-\zeta) \mathrm{t}^{p}+\zeta \mathrm{s}^{p}\right]^{\frac{1}{p}}\right) d \zeta .
$$

If $\mathfrak{U}$ is $(p, s)$-concave $F-I-V-F$, then Equation (35) is reversed.

Proof. Let $\mathfrak{U}$ be a $(p, s)$-convex $F-I-V$-F. Then, for each $\varphi \in[0,1]$, we have

$$
\begin{aligned}
& \mathfrak{U}_{*}\left(\left[\zeta \mathrm{t}^{p}\right.\right.\left.\left.+(1-\zeta) \mathrm{s}^{p}\right]^{\frac{1}{p}}, \varphi\right) \Psi\left(\left[\zeta \mathrm{t}^{p}+(1-\zeta) \mathrm{s}^{p}\right]^{\frac{1}{p}}\right) \\
& \leq\left(\zeta^{s} \mathfrak{U}_{*}(\mathrm{t}, \varphi)+(1-\zeta)^{s} \mathfrak{U}_{*}(\mathrm{~s}, \varphi)\right) \Psi\left(\left[\zeta \mathrm{t}^{p}+(1-\zeta) \mathrm{s}^{p}\right]^{\frac{1}{p}}\right), \\
& \mathfrak{U}^{*}\left(\left[\zeta \mathrm{t}^{p}+(1-\zeta) \mathrm{s}^{p}\right]^{\frac{1}{p}}, \varphi\right) \Psi\left(\left[\zeta \mathrm{t}^{p}+(1-\zeta) \mathrm{s}^{p}\right]^{\frac{1}{p}}\right) \\
& \leq\left(\zeta^{s} \mathfrak{U}^{*}(\mathrm{t}, \varphi)+(1-\zeta)^{s} \mathfrak{U}^{*}(\mathrm{~s}, \varphi)\right) \Psi\left(\left[\zeta \mathrm{t}^{p}+(1-\zeta) \mathrm{s}^{p}\right]^{\frac{1}{p}}\right) .
\end{aligned}
$$


and

$$
\begin{aligned}
& \mathfrak{U}_{*}\left(\left[(1-\zeta) \mathrm{t}^{p}+\zeta \mathrm{s}^{p}\right]^{\frac{1}{p}}, \varphi\right) \Psi\left(\left[(1-\zeta) \mathrm{t}^{p}+\zeta \mathrm{s}^{p}\right]^{\frac{1}{p}}\right) \\
& \quad \leq\left((1-\zeta)^{s} \mathfrak{U}_{*}(\mathrm{t}, \varphi)+\zeta^{s} \mathfrak{U}_{*}(\mathrm{~s}, \varphi)\right) \Psi\left(\left[(1-\zeta) \mathrm{t}^{p}+\zeta \mathrm{s}^{p}\right]^{\frac{1}{p}}\right), \\
& \mathfrak{U}^{*}\left(\left[(1-\zeta) \mathrm{t}^{p}+\zeta \mathrm{s}^{p}\right]^{\frac{1}{p}}, \varphi\right) \Psi\left(\left[(1-\zeta) \mathrm{t}^{p}+\zeta \mathrm{s}^{p}\right]^{\frac{1}{p}}\right) \\
& \leq\left((1-\zeta)^{s} \mathfrak{U}^{*}(\mathrm{t}, \varphi)+\zeta^{s} \mathfrak{U}^{*}(\mathrm{~s}, \varphi)\right) \Psi\left(\left[(1-\zeta) \mathrm{t}^{p}+\zeta \mathrm{s}^{p}\right]^{\frac{1}{p}}\right) .
\end{aligned}
$$

After adding Equations (36) and (37), and integrating over [0, 1], we get

$$
\begin{aligned}
& \int_{0}^{1} \mathfrak{U}_{*}\left(\left[\zeta t^{p}+(1-\zeta) \mathbf{s}^{p}\right]^{\frac{1}{p}}, \varphi\right) \Psi\left(\left[\zeta t^{p}+(1-\zeta) \mathbf{s}^{p}\right]^{\frac{1}{p}}\right) d \zeta \\
& +\int_{0}^{1} \mathfrak{U}_{*}\left(\left[(1-\zeta) \mathbf{t}^{p}+\zeta \mathbf{s}^{p}\right]^{\frac{1}{p}}, \varphi\right) \Psi\left(\left[(1-\zeta) \mathbf{t}^{p}+\zeta \mathbf{s}^{p}\right]^{\frac{1}{p}}\right) d \zeta \\
& \leq \int_{0}^{1}\left[\begin{array}{c}
\mathfrak{U}_{*}(\mathrm{t}, \varphi)\left\{\zeta^{s} \Psi\left(\left[\zeta t^{p}+(1-\zeta) \mathbf{s}^{p}\right]^{\frac{1}{p}}\right)+(1-\zeta)^{s} \Psi\left(\left[(1-\zeta) t^{p}+\zeta \mathbf{s}^{p}\right]^{\frac{1}{p}}\right)\right\} \\
+\mathfrak{U}_{*}(\mathrm{~s}, \varphi)\left\{(1-\zeta)^{s} \Psi\left(\left[\zeta \mathrm{t}^{p}+(1-\zeta) \mathbf{s}^{p}\right]^{\frac{1}{p}}\right)+\zeta^{s} \Psi\left(\left[(1-\zeta) t^{p}+\zeta \mathbf{s}^{p}\right]^{\frac{1}{p}}\right)\right\}
\end{array}\right] d \zeta, \\
& \int_{0}^{1} \mathfrak{U}^{*}\left(\left[\zeta \mathrm{t}^{p}+(1-\zeta) \mathrm{s}^{p}\right]^{\frac{1}{p}}, \varphi\right) \Psi\left(\left[\zeta \mathrm{t}^{p}+(1-\zeta) \mathrm{s}^{p}\right]^{\frac{1}{p}}\right) d \zeta \\
& +\int_{0}^{1} \mathfrak{U}^{*}\left(\left[(1-\zeta) \mathfrak{t}^{p}+\zeta \mathbf{s}^{p}\right]^{\frac{1}{p}}, \varphi\right) \Psi\left(\left[(1-\zeta) \mathbf{t}^{p}+\zeta \mathbf{s}^{p}\right]^{\frac{1}{p}}\right) d \zeta \\
& \leq \int_{0}^{1}\left[\begin{array}{c}
\mathfrak{U}^{*}(\mathrm{t}, \varphi)\left\{\zeta^{s} \Psi\left(\left[\zeta \mathrm{t}^{p}+(1-\zeta) \mathbf{s}^{p}\right]^{\frac{1}{p}}\right)+(1-\zeta)^{s} \Psi\left(\left[(1-\zeta) \mathbf{t}^{p}+\zeta \mathrm{s}^{p}\right]^{\frac{1}{p}}\right)\right\} \\
+\mathfrak{U}^{*}(\mathrm{~s}, \varphi)\left\{(1-\zeta)^{s} \Psi\left(\left[\zeta \mathrm{t}^{p}+(1-\zeta) \mathbf{s}^{p}\right]^{\frac{1}{p}}\right)+\zeta^{s} \Psi\left(\left[(1-\zeta) \mathbf{t}^{p}+\zeta \mathbf{s}^{p}\right]^{\frac{1}{p}}\right)\right\}
\end{array}\right] d \zeta . \\
& =2 \mathfrak{U}_{*}(\mathrm{t}, \varphi) \int_{0}^{1} \zeta^{s} \Psi\left(\left[\zeta \mathrm{t}^{p}+(1-\zeta) \mathrm{s}^{p}\right]^{\frac{1}{p}}\right) d \zeta+2 \mathfrak{U}_{*}(\mathrm{~s}, \varphi) \int_{0}^{1} \zeta^{s} \Psi\left(\left[(1-\zeta) \mathrm{t}^{p}+\zeta \mathrm{s}^{p}\right]^{\frac{1}{p}}\right) d \zeta \\
& =2 \mathfrak{U}^{*}(\mathrm{t}, \varphi) \int_{0}^{1} \zeta^{s} \Psi\left(\left[\zeta \mathrm{t}^{p}+(1-\zeta) \mathbf{s}^{p}\right]^{\frac{1}{p}}\right) d \zeta+2 \mathfrak{U}^{*}(\mathrm{~s}, \varphi) \int_{0}^{1} \zeta^{s} \Psi\left(\left[(1-\zeta) \mathbf{t}^{p}+\zeta \mathbf{s}^{p}\right]^{\frac{1}{p}}\right) d \zeta \text {. }
\end{aligned}
$$

Since $\Psi$ is symmetric, then

$$
\begin{aligned}
& =2\left[\mathfrak{U}_{*}(\mathrm{t}, \varphi)+\mathfrak{U}_{*}(\mathrm{~s}, \varphi)\right] \int_{0}^{1} \zeta^{s} \Psi\left(\left[(1-\zeta) \mathrm{t}^{p}+\zeta \mathbf{s}^{p}\right]^{\frac{1}{p}}\right) d \zeta \\
& =2\left[\mathfrak{U}^{*}(\mathrm{t}, \varphi)+\mathfrak{U}^{*}(\mathrm{~s}, \varphi)\right] \int_{0}^{1} \zeta^{s} \Psi\left(\left[(1-\zeta) \mathrm{t}^{p}+\zeta \mathrm{s}^{p}\right]^{\frac{1}{p}}\right) d \zeta .
\end{aligned}
$$

Since

$$
\begin{aligned}
& \int_{0}^{1} \mathfrak{U}_{*}\left(\left[\zeta t^{p}+(1-\zeta) \mathbf{s}^{p}\right]^{\frac{1}{p}}, \varphi\right) \Psi\left(\left[\zeta t^{p}+(1-\zeta) \mathbf{s}^{p}\right]^{\frac{1}{p}}\right) d \zeta \\
& =\int_{0}^{1} \mathfrak{U}_{*}\left(\left[(1-\zeta) \mathfrak{t}^{p}+\zeta \mathbf{s}^{p}\right]^{\frac{1}{p}}, \varphi\right) \Psi\left(\left[(1-\zeta) \mathfrak{t}^{p}+\zeta \mathbf{s}^{p}\right]^{\frac{1}{p}}\right) d \zeta \\
& =\frac{p}{s^{p}-t^{p}} \int_{\mathrm{t}}^{s} \varkappa^{p-1} \mathfrak{U}_{*}(\varkappa, \varphi) \Psi(\varkappa) d \varkappa \text {, } \\
& \int_{0}^{1} \mathfrak{U}^{*}\left(\left[\zeta \mathfrak{t}^{p}+(1-\zeta) \mathbf{s}^{p}\right]^{\frac{1}{p}}, \varphi\right) \Psi\left(\left[\zeta \mathfrak{t}^{p}+(1-\zeta) \mathbf{s}^{p}\right]^{\frac{1}{p}}\right) d \zeta \\
& =\int_{0}^{1} \mathfrak{U}^{*}\left(\left[(1-\zeta) \mathbf{t}^{p}+\zeta \mathbf{s}^{p}\right]^{\frac{1}{p}}, \varphi\right) \Psi\left(\left[(1-\zeta) \mathbf{t}^{p}+\zeta \mathbf{s}^{p}\right]^{\frac{1}{p}}\right) d \zeta \\
& =\frac{p}{\mathrm{~s}^{p}-\mathrm{t}^{p}} \int_{\mathrm{t}}^{\mathrm{s}} \varkappa^{p-1} \mathfrak{U}^{*}(\varkappa, \varphi) \Psi(\varkappa) d \varkappa \text {, }
\end{aligned}
$$

From Equation (39) and integrating with respect to $\zeta$ over $[0,1]$, we have

$$
\begin{aligned}
& \frac{p}{\mathrm{~s}^{p}-\mathrm{t}^{p}} \int_{\mathrm{t}}^{\mathrm{s}} \varkappa^{p-1} \mathfrak{U}_{*}(\varkappa, \varphi) \Psi(\varkappa) d \varkappa \leq\left[\mathfrak{U}_{*}(\mathrm{t}, \varphi)+\mathfrak{U}_{*}(\mathrm{~s}, \varphi)\right] \int_{0}^{1} \zeta^{s} \Psi\left(\left[(1-\zeta) \mathrm{t}^{p}+\zeta \mathbf{s}^{p}\right]^{\frac{1}{p}}\right) d \zeta, \\
& \frac{p}{\mathrm{~s}^{p}-\mathrm{t}^{p}} \int_{\mathrm{t}}^{\mathrm{s}} \varkappa^{p-1} \mathfrak{U}^{*}(\varkappa, \varphi) \Psi(\varkappa) d \varkappa \leq\left[\mathfrak{U}^{*}(\mathrm{t}, \varphi)+\mathfrak{U}^{*}(\mathrm{~s}, \varphi)\right] \int_{0}^{1} \zeta^{s} \Psi\left(\left[(1-\zeta) \mathbf{t}^{p}+\zeta \mathrm{s}^{p}\right]^{\frac{1}{p}}\right) d \zeta,
\end{aligned}
$$

that is,

$$
\begin{aligned}
\frac{p}{\mathrm{~s}^{p}-\mathrm{t}^{p}}[ & \left.\int_{\mathrm{t}}^{\mathrm{s}} \varkappa^{p-1} \mathfrak{U}_{*}(\varkappa, \varphi) \Psi(\varkappa) d \varkappa, \quad \int_{\mathrm{t}}^{\mathrm{s}} \varkappa^{p-1} \mathfrak{U}^{*}(\varkappa, \varphi) \Psi(\varkappa) d \varkappa\right] \\
& \leq_{I}\left[\mathfrak{U}_{*}(\mathrm{t}, \varphi)+\mathfrak{U}_{*}(\mathrm{~s}, \varphi), \mathfrak{U}^{*}(\mathrm{t}, \varphi)+\mathfrak{U}^{*}(\mathrm{~s}, \varphi)\right] \int_{0}^{1} \zeta^{s} \Psi\left(\left[(1-\zeta) \mathrm{t}^{p}+\zeta \mathrm{s}^{p}\right]^{\frac{1}{p}}\right) d \zeta,
\end{aligned}
$$


hence

$$
\frac{p}{\mathrm{~s}^{p}-\mathrm{t}^{p}}(F R) \int_{\mathrm{t}}^{\mathrm{s}} \varkappa^{p-1} \mathfrak{U}(\varkappa) \Psi(\varkappa) d \varkappa \preccurlyeq[\mathfrak{U}(\mathrm{t}) \widetilde{+} \mathfrak{U}(\mathrm{s})] \int_{0}^{1} \zeta^{s} \Psi\left(\left[(1-\zeta) \mathrm{t}^{p}+\zeta \mathrm{s}^{p}\right]^{\frac{1}{p}}\right) d \zeta .
$$

Theorem 9. (First $\mathrm{H}-\mathrm{H}$ Fejér inequality for $(p, s)$-convex $F-I-V-F)$ Let $\mathfrak{U}:[\mathrm{t}, \mathrm{s}] \rightarrow \mathbb{F}_{C}(\mathbb{R})$ be a $(p, s)$-convex $F-I-V-F$ with $\mathrm{t}<\mathrm{s}$, whose $\varphi$-levels define the family of $I-V-F \mathrm{~s}$ $\mathfrak{U}_{\varphi}:[\mathrm{t}, \mathrm{s}] \subset \mathbb{R} \rightarrow \mathcal{K}_{C}{ }^{+}$are given by $\mathfrak{U}_{\varphi}(\varkappa)=\left[\mathfrak{U}_{*}(\varkappa, \varphi), \mathfrak{U}^{*}(\varkappa, \varphi)\right]$ for all $\in[\mathrm{t}, \mathrm{s}]$ and for all $\varphi \in[0,1]$. If $\mathfrak{U} \in \mathcal{F} \mathcal{R}_{([\mathrm{t}, \mathrm{s}])}$ and $\Psi:[\mathrm{t}, \mathrm{s}] \rightarrow \mathbb{R}, \Psi(\varkappa) \geq 0, p$-symmetric with respect to $\left[\frac{\mathrm{t}^{p}+\mathrm{s}^{p}}{2}\right]^{\frac{1}{p}}$, and $\int_{\mathrm{t}}^{\mathrm{s}} \Psi(\varkappa) d \varkappa>0$, then

$$
2^{s-1} \mathfrak{U}\left(\left[\frac{\mathrm{t}^{p}+\mathrm{s}^{p}}{2}\right]^{\frac{1}{p}}\right) \preccurlyeq \frac{p}{\int_{\mathrm{t}}^{\mathrm{s}} \varkappa^{p-1} \Psi(\varkappa) d \varkappa}(F R) \int_{\mathrm{t}}^{\mathrm{s}} \varkappa^{p-1} \mathfrak{U}(\varkappa) \Psi(\varkappa) d \varkappa .
$$

If $\mathfrak{U}$ is $(p, s)$-concave $F-I-V-F$, then inequality (40) is reversed.

Proof. Since $\mathfrak{U}$ is a $(p, s)$-convex $F-I-V-F$, then, for each $\varphi \in[0,1]$, we have

$$
\begin{aligned}
& 2^{s} \mathfrak{U}_{*}\left(\left[\frac{\mathrm{t}^{p}+\mathrm{s}^{p}}{2}\right]^{\frac{1}{p}}, \varphi\right) \leq \mathfrak{U}_{*}\left(\left[\zeta \mathrm{t}^{p}+(1-\zeta) \mathbf{s}^{p}\right]^{\frac{1}{p}}, \varphi\right)+\mathfrak{U}_{*}\left(\left[(1-\zeta) \mathrm{t}^{p}+\zeta \mathrm{s}^{p}\right]^{\frac{1}{p}}, \varphi\right), \\
& 2^{s} \mathfrak{U}^{*}\left(\left[\frac{\mathrm{t}^{p}+\mathrm{s}^{p}}{2}\right]^{\frac{1}{p}}, \varphi\right) \leq \mathfrak{U}^{*}\left(\left[\zeta \mathrm{t}^{p}+(1-\zeta) \mathbf{s}^{p}\right]^{\frac{1}{p}}, \varphi\right)+\mathfrak{U}^{*}\left(\left[(1-\zeta) \mathrm{t}^{p}+\zeta \mathbf{s}^{p}\right]^{\frac{1}{p}}, \varphi\right) .
\end{aligned}
$$

By multiplying Equation (41) by $\Psi\left(\left[\zeta t^{p}+(1-\zeta) s^{p}\right]^{\frac{1}{p}}\right)=\Psi\left(\left[(1-\zeta) t^{p}+\zeta s^{p}\right]^{\frac{1}{p}}\right)$ and integrating it by $\zeta$ over $[0,1]$, we obtain

$$
\begin{aligned}
& 2^{s} \mathfrak{U}_{*}\left(\left[\frac{\mathrm{t}^{p}+\mathrm{s}^{p}}{2}\right]^{\frac{1}{p}}, \varphi\right) \int_{0}^{1} \Psi\left(\left[(1-\zeta) \mathrm{t}^{p}+\zeta \mathrm{s}^{p}\right]^{\frac{1}{p}}, \varphi\right) d \zeta \\
& \leq\left(\begin{array}{c}
\int_{0}^{1} \mathfrak{U}_{*}\left(\left[\zeta t^{p}+(1-\zeta) \mathbf{s}^{p}\right]^{\frac{1}{p}}, \varphi\right) \Psi\left(\left[\zeta t^{p}+(1-\zeta) \mathbf{s}^{p}\right]^{\frac{1}{p}}\right) d \zeta \\
+\int_{0}^{1} \mathfrak{U}_{*}\left(\left[(1-\zeta) t^{p}+\zeta \mathbf{s}^{p}\right]^{\frac{1}{p}}, \varphi\right) \Psi\left(\left[(1-\zeta) t^{p}+\zeta \mathbf{s}^{p}\right]^{\frac{1}{p}}\right) d \zeta
\end{array}\right), \\
& 2^{\mathrm{s}} \mathfrak{U}^{*}\left(\left[\frac{\mathrm{t}^{p}+\mathrm{s}^{p}}{2}\right]^{\frac{1}{p}}, \varphi\right) \int_{0}^{1} \Psi\left(\left[(1-\zeta) \mathbf{t}^{p}+\zeta \mathbf{s}^{p}\right]^{\frac{1}{p}}\right) d \zeta \\
& \leq\left(\begin{array}{c}
\int_{0}^{1} \mathfrak{U}^{*}\left(\left[\zeta \mathfrak{t}^{p}+(1-\zeta) \mathbf{s}^{p}\right]^{\frac{1}{p}}, \varphi\right) \Psi\left(\left[\zeta t^{p}+(1-\zeta) \mathbf{s}^{p}\right]^{\frac{1}{p}}\right) d \zeta \\
+\int_{0}^{1} \mathfrak{U}^{*}\left(\left[(1-\zeta) \mathfrak{t}^{p}+\zeta \mathbf{s}^{p}\right]^{\frac{1}{p}}, \varphi\right) \Psi\left(\left[(1-\zeta) \mathfrak{t}^{p}+\zeta \mathbf{s}^{p}\right]^{\frac{1}{p}}\right) d \zeta
\end{array}\right) .
\end{aligned}
$$

Since

$$
\begin{aligned}
& \int_{0}^{1} \mathfrak{U}_{*}\left(\left[\zeta \mathbf{t}^{p}+(1-\zeta) \mathbf{s}^{p}\right]^{\frac{1}{p}}, \varphi\right) \Psi\left(\left[\zeta t^{p}+(1-\zeta) \mathbf{s}^{p}\right]^{\frac{1}{p}}\right) d \zeta \\
& =\int_{0}^{1} \mathfrak{U}_{*}\left(\left[(1-\zeta) \mathbf{t}^{p}+\zeta \mathbf{s}^{p}\right]^{\frac{1}{p}}, \varphi\right) \Psi\left(\left[(1-\zeta) \mathbf{t}^{p}+\zeta \mathbf{s}^{p}\right]^{\frac{1}{p}}\right) d \zeta \\
& =\frac{p}{\mathrm{~s}^{p}-\mathrm{t}^{p}} \int_{\mathrm{t}}^{\mathrm{s}} \varkappa^{p-1} \mathfrak{U}_{*}(\varkappa, \varphi) \Psi(\varkappa) d \varkappa, \\
& \int_{0}^{1} \mathfrak{U}^{*}\left(\left[\zeta \mathfrak{t}^{p}+(1-\zeta) \mathbf{s}^{p}\right]^{\frac{1}{p}}, \varphi\right) \Psi\left(\left[\zeta \mathbf{t}^{p}+(1-\zeta) \mathbf{s}^{p}\right]^{\frac{1}{p}}\right) d \zeta \\
& =\int_{0}^{1} \mathfrak{U}^{*}\left(\left[(1-\zeta) \mathbf{t}^{p}+\zeta \mathbf{s}^{p}\right]^{\frac{1}{p}}, \varphi\right) \Psi\left(\left[(1-\zeta) \mathbf{t}^{p}+\zeta \mathbf{s}^{p}\right]^{\frac{1}{p}}\right) d \zeta \\
& =\frac{p}{\mathrm{~s}^{p}-\mathrm{t}^{p}} \int_{\mathrm{t}}^{\mathrm{s}} \varkappa^{p-1} \mathfrak{U}^{*}(\varkappa, \varphi) \Psi(\varkappa) d \varkappa \text {, }
\end{aligned}
$$


From Equation (43), we have

$$
\begin{aligned}
& 2^{s-1} \mathfrak{U}_{*}\left(\left[\frac{\mathrm{t}^{p}+\mathrm{s}^{p}}{2}\right]^{\frac{1}{p}}, \varphi\right) \leq \frac{p}{\int_{\mathrm{t}}^{\mathrm{s}} \Psi(\varkappa) d \varkappa} \int_{\mathrm{t}}^{\mathrm{s}} \varkappa^{p-1} \mathfrak{U}_{*}(\varkappa, \varphi) \Psi(\varkappa) d \varkappa, \\
& 2^{s-1} \mathfrak{U}^{*}\left(\left[\frac{\mathrm{t}^{p}+\mathrm{s}^{p}}{2}\right]^{\frac{1}{p}}, \varphi\right) \leq \frac{p}{\int_{\mathrm{t}}^{\mathrm{s}} \Psi(\varkappa) d \varkappa} \int_{\mathrm{t}}^{\mathrm{s}} \varkappa^{p-1} \mathfrak{U}^{*}(\varkappa, \varphi) \Psi(\varkappa) d \varkappa .
\end{aligned}
$$

From this, we have

$$
\begin{gathered}
2^{s-1}\left[\mathfrak{U}_{*}\left(\left[\frac{\mathrm{t}^{p}+\mathrm{s}^{p}}{2}\right]^{\frac{1}{p}}, \varphi\right), \mathfrak{U}^{*}\left(\left[\frac{\mathrm{t}^{p}+\mathrm{s}^{p}}{2}\right]^{\frac{1}{p}}, \varphi\right)\right] \\
\leq_{I} \frac{p}{\int_{\mathrm{t}}^{\mathrm{s}} \Psi(\varkappa) d \varkappa}\left[\int_{\mathrm{t}}^{\mathrm{s}} \varkappa^{p-1} \mathfrak{U}_{*}(\varkappa, \varphi) \Psi(\varkappa) d \varkappa, \int_{\mathrm{t}}^{\mathrm{s}} \varkappa^{p-1} \mathfrak{U}^{*}(\varkappa, \varphi) \Psi(\varkappa) d \varkappa\right],
\end{gathered}
$$

that is

$$
2^{s-1} \mathfrak{U}\left(\left[\frac{\mathrm{t}^{p}+\mathrm{s}^{p}}{2}\right]^{\frac{1}{p}}\right) \preccurlyeq \frac{p}{\int_{\mathrm{t}}^{\mathrm{s}} \varkappa^{p-1} \Psi(\varkappa) d \varkappa}(F R) \int_{\mathrm{t}}^{\mathrm{s}} \varkappa^{p-1} \mathfrak{U}(\varkappa, \varphi) \Psi(\varkappa) d \varkappa,
$$

and this completes the proof.

Remark 5. If we attempt to take $s=1$ in Theorems 8 and 9 , then we achieve the appropriate theorems for $p$-convex F-I-V-Fs, see [13]:

- $\quad$ If we attempt to take $\mathfrak{U}_{*}(\varkappa, \varphi)=\mathfrak{U}^{*}(\varkappa, \varphi)$ with $\varphi=1$, then, from Theorems 8 and 9, we achieve classical first and second $\mathrm{H}-\mathrm{H}$ Fejér inequality for $(p, s)$-convex function, [21];

- If in Theorems 8 and 9, we attempt to take $\mathfrak{U}_{*}(\varkappa, \varphi)=\mathfrak{U}^{*}(\varkappa, \varphi)$ with $\varphi=1$ and $s=1$, then we acquire the classical appropriate theorems for $p$-convex function, see [49];

- If, in Theorems 8 and 9, we attempt to take $\mathfrak{U}_{*}(\varkappa, \varphi)=\mathfrak{U}^{*}(\varkappa, \varphi)$ with $\varphi=1, s=1$ and $p=1$, then we acquire the appropriate theorems for a convex function [48];

- If we attempt to take $\Psi(\varkappa)=1$, then combining Theorem 8 and Theorem 9 , we acquire Theorem 4.1.

Example 4. We consider the $F-I-V-F \mathfrak{U}:[1,4] \rightarrow \mathbb{F}_{C}(\mathbb{R})$ defined by

$$
\mathfrak{U}(\varkappa)(\sigma)=\left\{\begin{array}{cc}
\frac{\sigma-e^{\varkappa p}}{e^{\varkappa p}}, & \sigma \in\left[e^{\varkappa p}, 2 e^{\varkappa p}\right] \\
\frac{4 e^{\varkappa p}-\sigma}{2 e^{\varkappa p}}, & \sigma \in\left(2 e^{\varkappa p}, 4 e^{\varkappa p}\right] \\
0, & \text { otherwise }
\end{array}\right.
$$

Then, for each $\varphi \in[0,1]$, we have $\mathfrak{U}_{\varphi}(\varkappa)=\left[(1+\varphi) e^{\varkappa p}, 2(2-\varphi) e^{\varkappa p}\right]$. Since end point functions $\mathfrak{U}_{*}(\varkappa, \varphi), \mathfrak{U}^{*}(\varkappa, \varphi)$ are $(p, s)$-convex functions, for each $s, \varphi \in[0,1]$, then $\mathfrak{U}(\varkappa)$ is $(p, s)$-convex $F-I-V-F$. If

$$
\Psi(\varkappa)=\left\{\begin{array}{cc}
\varkappa^{p}-1, & \sigma \in\left[1, \frac{5}{2}\right] \\
4-\varkappa^{p}, & \sigma \in\left(\frac{5}{2}, 4\right]
\end{array}\right.
$$

where $p=1$. Then, we have

$$
\begin{array}{r}
\frac{p}{\mathrm{~s}^{p}-\mathrm{t}^{p}} \int_{1}^{4} \varkappa^{p-1} \mathfrak{U}_{*}(\varkappa, \varphi) \Psi(\varkappa) d \varkappa=\frac{1}{3} \int_{1}^{4} \varkappa^{p-1} \mathfrak{U}_{*}(\varkappa, \varphi) \Psi(\varkappa) d \varkappa \\
=\frac{1}{3} \int_{1}^{\frac{5}{2}} \varkappa^{p-1} \mathfrak{U}_{*}(\varkappa, \varphi) \Psi(\varkappa) d \varkappa+\frac{1}{3} \int_{\frac{5}{2}}^{4} \varkappa^{p-1} \mathfrak{U}_{*}(\varkappa, \varphi) \Psi(\varkappa) d \varkappa \\
=\frac{1}{3}(1+\varphi) \int_{1}^{\frac{5}{2}} e(-1) d \varkappa+\frac{1}{3}(1+\varphi) \int_{\frac{5}{2}}^{4} e(4-) d \varkappa \approx 11(1+\varphi), \\
\frac{p}{\mathrm{~s}^{p}-\mathrm{t}^{p}} \int_{1}^{4} \varkappa^{p-1} \mathfrak{U}^{*}(\varkappa, \varphi) \Psi(\varkappa) d \varkappa=\frac{1}{3} \int_{1}^{4} \varkappa^{p-1} \mathfrak{U}^{*}(\varkappa, \varphi) \Psi(\varkappa) d \varkappa \\
=\frac{1}{3} \int_{1}^{\frac{5}{2}} \varkappa^{p-1} \mathfrak{U}^{*}(\varkappa, \varphi) \Psi(\varkappa) d \varkappa+\frac{1}{3} \int_{\frac{5}{2}}^{4} \varkappa^{p-1} \mathfrak{U}^{*}(\varkappa, \varphi) \Psi(\varkappa) d \varkappa \\
=\frac{2}{3}(2-\varphi) \int_{1}^{\frac{5}{2}} e(-1) d \varkappa+\frac{2}{3}(2-\varphi) \int_{\frac{5}{2}}^{4} e(4-) d \varkappa \approx 22(2-\varphi),
\end{array}
$$


and

$$
\begin{aligned}
{\left[\mathfrak{U}^{*}(\mathrm{t}, \varphi)\right.} & \left.+\mathfrak{U}^{*}(\mathrm{~s}, \varphi)\right] \int_{0}^{1} \zeta^{s} \Psi\left(\left[(1-\tau) \mathrm{t}^{p}+\tau \mathrm{s}^{p}\right]^{\frac{1}{p}}\right) d \tau \\
{\left[\mathfrak{U}_{*}(\mathrm{t}, \varphi)\right.} & \left.+\mathfrak{U}_{*}(\mathrm{~s}, \varphi)\right] \int_{0}^{1} \zeta^{s} \Psi\left(\left[(1-\tau) \mathrm{t}^{p}+\tau \mathbf{s}^{p}\right]^{\frac{1}{p}}\right) d \tau \\
& =(1+\varphi)\left[e+e^{4}\right]\left[\int_{0}^{\frac{1}{2}} 3 \tau^{2} d+\int_{\frac{1}{2}}^{1} \tau(3-3 \tau) d \tau\right] \approx \frac{43}{2}(1+\varphi) \\
& =2(2-\varphi)\left[e+e^{4}\right]\left[\int_{0}^{\frac{1}{2}} 3 \tau^{2} d+\int_{\frac{1}{2}}^{1} \tau(3-3 \tau) d \tau\right] \approx 43(2-\varphi) .
\end{aligned}
$$

From Equations (46) and (47), we have

$$
[11(1+\varphi), 22(2-\varphi)] \leq I\left[\frac{43}{2}(1+\varphi), 43(2-\varphi)\right], \text { for each } \varphi \in[0,1]
$$

Hence, Theorem 8 is verified.

For Theorem 9, we have

$$
\begin{gathered}
2^{s-1} \mathfrak{U}_{*}\left(\left[\frac{\mathrm{t}^{p}+\mathrm{s}^{p}}{2}\right]^{\frac{1}{p}}, \varphi\right) \approx \frac{61}{5}(1+\varphi), \\
2^{s-1} \mathfrak{U}^{*}\left(\left[\frac{\mathrm{t}^{p}+\mathrm{s}^{p}}{2}\right]^{\frac{1}{p}}, \varphi\right) \approx \frac{122}{5}(2-\varphi), \\
\int_{\mathrm{t}}^{s} \varkappa^{p-1} \Psi(\varkappa) d \varkappa=\int_{1}^{\frac{5}{2}}(\varkappa-1) d \varkappa \int_{\frac{5}{2}}^{4}(4-) d \varkappa=\frac{9}{4}, \\
\frac{p}{\int_{\mathrm{t}}^{s} \varkappa^{p-1 \Psi(\varkappa) d \varkappa}} \int_{1}^{4} \varkappa^{p-1} \mathfrak{U}_{*}(\varkappa, \varphi) \Psi(\varkappa) d \varkappa \approx \frac{73}{5}(1+\varphi), \\
\frac{p}{\int_{\mathrm{t}}^{s} \varkappa^{p-1 \Psi(\varkappa) d \varkappa}} \int_{1}^{4} \varkappa^{p-1} \mathfrak{U}^{*}(\varkappa, \varphi) \Psi(\varkappa) d \varkappa \approx \frac{293}{10}(2-\varphi) .
\end{gathered}
$$

From Equations (48) and (49), we have

$$
\left[\frac{61}{5}(1+\varphi), \frac{122}{5}(2-\varphi)\right] \leq I\left[\frac{73}{5}(1+\varphi), \frac{293}{10}(2-\varphi)\right] .
$$

Hence, Theorem 9 has been demonstrated.

\section{Conclusions and Future Developments}

Through this study, we have provided a reformative version of the different inequalities in the frame of fuzzy interval space, which offers a better approximation than the interval integral inequalities.

Then, for mappings satisfying the property "the product of two $(p, s)$-convex $F-I-V-F s$ is a $(p, s)$-convex $F-I-V-F$ ", we created certain fuzzy interval integral inequalities in terms of the fuzzy interval $\mathrm{H}-\mathrm{H}$ type inequalities. It is a fascinating topic to apply these fuzzy interval inequalities to $\varphi$-type special means, numerical integration, and probability density functions. With the methods and ideas provided in this article, the interested readers are encouraged to further excavation on fuzzy interval inequalities. In the future, we will try to explore this concept and its generalizations with the help of fuzzy fractional integral operators.

Author Contributions: Conceptualization, M.B.K.; validation, H.B. and S.T.; formal analysis, H.B. and S.T.; investigation, M.B.K. and S.T.; resources, M.B.K. and H.B.; writing-original draft, M.B.K. and H.B.; writing-review and editing, M.B.K. and S.T.; visualization, M.B.K., H.B. and S.T.; supervision, M.B.K. and S.T.; project administration, H.B., M.B.K. and S.T. All authors have read and agreed to the published version of the manuscript.

Funding: This research received no external funding. 
Institutional Review Board Statement: Not applicable.

Informed Consent Statement: Not applicable.

Data Availability Statement: Not Applicable.

Acknowledgments: The authors would like to thank the Rector, COMSATS University Islamabad, Islamabad, Pakistan, for providing excellent research.

Conflicts of Interest: The authors declare no conflict of interest.

$\begin{array}{ll}\text { Abbreviations } & \\ \mathcal{K}_{C} & \text { Collection of all closed and bounded intervals } \\ \mathcal{K}_{C}^{+} & \text {Collection of all closed and bounded positive intervals } \\ \mathbb{F}_{C}(\mathbb{R}) & \text { Collection of all closed and bounded fuzzy intervals } \\ F-I-V-F \mathrm{~s} & \text { Fuzzy-interval-valued functions } \\ I-V-F \mathrm{~s} & \text { Interval-valued functions } \\ \leq_{I} & \text { order relation } \\ \preccurlyeq & \text { fuzzy order relation } \\ (p, s) \text {-convex } F-I-V-F s & (p, s) \text {-Convex fuzzy-interval-valued functions } \\ \mathrm{H}-\mathrm{H} \text { inequality } & \text { Hermite-Hadamard inequality } \\ \mathrm{H}-\mathrm{H} \text { Fejér inequality } & \text { Hermite-Hadamard-Fejér inequality } \\ (F R) \text {-integrable } & \text { Fuzzy Riemann integrable } \\ \mathcal{R} & \text { Riemann integrable real-valued functions } \\ \mathcal{I} \mathcal{R}_{[\mathrm{t}, \mathrm{s}]} & \text { Riemann integrable } I-V-F \mathrm{~s} \\ \mathcal{F} \mathcal{R}([\mathrm{t}, \mathrm{s}]) & \text { Riemann integrable } F-I-V \text { - } F \mathrm{~s}\end{array}$

\section{References}

1. Dragomir, S.S.; Pearce, V. Selected Topics on Hermite-Hadamard Inequalities and Applications; RGMIA Monographs: Victoria, Australia, 2000.

2. Mehrez, K.; Agarwal, P. New Hermite-Hadamard type integral inequalities for convex functions and their applications. J. Comput. Appl. Math. 2019, 50, 274-285. [CrossRef]

3. Mitrinović, D.S.; Pečarić, J.E.; Fink, A.M. Classical and New Inequalities in Analysis. Mathematics and Its Applications; (East European Series); Kluwer Academic Publishers Group: Dordrecht, The Netherlands, 1993; Volume 61.

4. Awan, M.U.; Akhtar, N.; Iftikhar, S.; Noor, M.A.; Chu, Y.-M. New Hermite-Hadamard type inequalities for n-polynomial harmonically convex functions. J. Inequal. Appl. 2020, 2020, 125. [CrossRef]

5. Latif, M.A.; Rashid, S.; Dragomir, S.S.; Chu, Y.-M. Hermite-Hadamard type inequalities for co-ordinated convex and quasi-convex functions and their applications. J. Inequal. Appl. 2019, 2019, 317. [CrossRef]

6. Chu, Y.-M.; Xia, W.-F.; Zhang, X.-H. The Schur concavity, Schur multiplicative and harmonic convexities of the second dual form of the Hamy symmetric function with applications. J. Multivar. Anal. 2012, 105, 412-442. [CrossRef]

7. Ullah, S.Z.; Khan, M.A.; Khan, Z.A.; Chu, Y.-M. Integral majorization type inequalities for the functions in the sense of strong convexity. J. Funct. Spaces 2019, 2019, 9487823.

8. Ullah, S.Z.; Khan, M.A.; Chu, Y.-M. Majorization theorems for strongly convex functions. J. Inequal. Appl. 2019, $2019,58$.

9. Zhang, K.-S.; Wan, J.-P. P-convex functions and their properties. Pure Appl. Math. 2007, 23, 130-133.

10. Chang, S.S.; Zhu, Y.G. On variational inequalities for fuzzy mappings. Fuzzy Sets Syst. 1989, 32, 359-367. [CrossRef]

11. Nanda, S.; Kar, K. Convex fuzzy mappings. Fuzzy Sets Syst. 1992, 48, 129-132. [CrossRef]

12. Noor, M.A. Fuzzy preinvex functions. Fuzzy Sets Syst. 1994, 64, 95-104. [CrossRef]

13. Khan, M.B.; Noor, M.A.; Noor, K.I.; Chu, Y.-M. New Hermite-Hadamard Type Inequalities for-Convex Fuzzy-Interval-Valued Functions. Adv. Diff. Equ. 2021, 2021, 6-20. [CrossRef]

14. Liu, W. New integral inequalities involving beta function via P-convexity. Miskolc Math. Notes 2014, 15, 585-591. [CrossRef]

15. Sawano, Y.; Wadade, H. On the Gagliardo-Nirenberg type inequality in the critical Sobolev-Orrey space. J. Fourier Anal. Appl. 2013, 19, 20-47. [CrossRef]

16. Cowling, P.C.M.G.; Ricci, F. Hardy and uncertainty inequalities on stratified Lie groups. Adv. Math. 2015, 277, 365-387.

17. Gavrea, B.; Gavrea, I. On some Ostrowski type inequalities. Gen. Math. 2010, 18, 33-44.

18. Gunawan, H. Fractional integrals and generalized Olsen inequalities. Kyungpook Math. J. 2009, 49, 31-39. [CrossRef]

19. Hadamard, J. Étude sur les propriétés des fonctions entières en particulier d'une fonction considérée par Riemann. J. Math. Pure Appl. 1893, 58, 171-215.

20. Khan, M.B.; Treanță, S.; Soliman, M.S.; Nonlaopon, K.; Zaini, H.G. Some Hadamard-Fejér Type Inequalities for LR-Convex Interval-Valued Functions. Fractal Fract. 2022, 6, 6. [CrossRef]

21. Fang, Z.-B.; Shi, R.-J. On the ( $p, h)$-convex function and some integral inequalities. J. Inequal. Appl. 2014, 2014, 45. [CrossRef] 
22. Khan, M.B.; Zaini, H.G.; Treanță, S.; Soliman, M.S.; Nonlaopon, K. Riemann-Liouville Fractional Integral Inequalities for Generalized Pre-Invex Functions of Interval-Valued Settings Based upon Pseudo Order Relation. Mathematics 2022, 10, 204. [CrossRef]

23. Kara, H.; Ali, M.A.; Budak, H. Hermite-Hadamard-type inequalities for interval-valued coordinated convex functions involving generalized fractional integrals. Math. Methods Appl. Sci. 2021, 44, 104-123. [CrossRef]

24. Liu, P.; Khan, M.B.; Noor, M.A.; Noor, K.I. New Hermite-Hadamard and Jensen inequalities for log-s-convex fuzzy-intervalvalued functions in the second sense. Complex. Intell. Syst. 2021, 2021, 155. [CrossRef]

25. Khan, M.B.; Noor, M.A.; Abdullah, L.; Chu, Y.-M. Some New Classes of Preinvex Fuzzy-Interval-Valued Functions and Inequalities Int. J. Comput. Intell. Syst. 2021, 14, 1403-1418. [CrossRef]

26. Sarikaya, M.Z.; Set, E.; Yaldiz, H.; BaSak, N. Hermite-Hadamard's inequalities for fractional integrals and related fractional inequalities. Math. Comput. Model. 2013, 57, 2403-2407. [CrossRef]

27. Toplu, T.; Set, E.; Scan, I.; Maden, S. Hermite-Hadamard type inequalities for p-convex functions via Katugampola fractional integrals. Facta Univ. Ser. Math. Inform. 2019, 34, 149-164. [CrossRef]

28. Bede, B. Mathematics of fuzzy sets and fuzzy logic. Stud. Fuzziness Soft Comput. 2013, 295, 1-288.

29. Thaiprayoon, C.; Ntouyas, S.K.; Tariboon, J. On the nonlocal Katugampola fractional integral conditions for fractional Langevin equation. Adv. Diff. Equ. 2015, 2015, 374. [CrossRef]

30. RAgarwal, P.; Baleanu, D.; Nieto, J.J.; Torres, D.F.M.; Zhou, Y. A survey on fuzzy fractional differential and optimal control nonlocal evolution equations. J. Comput. Appl. Math. 2018, 339, 3-29. [CrossRef]

31. Ahmad, M.Z.; Hasan, M.K.; de Baets, B. Analytical and numerical solutions of fuzzy differential equations. Inf. Sci. 2013, 236, 156-167. [CrossRef]

32. Alijani, Z.; Baleanu, D.; Shiri, B.; Wu, G.C. Spline collocation methods for systems of fuzzy fractional differential equations. Chaos Solitons Fract. 2020, 131, 109510. [CrossRef]

33. Bede, B.; Gal, S.G. Generalizations of the differentiability of fuzzy-number-valued functions with applications to fuzzy differential equations. Fuzzy Sets Syst. 2005, 151, 581-599. [CrossRef]

34. Bede, B.; Stefanini, L. Generalized differentiability of fuzzy-valued functions. Fuzzy Sets Syst. 2013, 230, 119-141. [CrossRef]

35. Diamond, P.; Kloeden, P.E. Metric Spaces of Fuzzy Sets: Theory and Applications; World Scientific: Singapore, 1994.

36. Goetschel, R., Jr.; Voxman, W. Elementary fuzzy calculus. Fuzzy Sets Syst. 1986, 18, 31-43. [CrossRef]

37. Hoa, N.V. On the initial value problem for fuzzy differential equations of non-integer order $\alpha \in(1,2)$. Soft Comput. 2020, 24, 935-954. [CrossRef]

38. Mazandarani, M.; Najariyan, M. Type-2 fuzzy fractional derivatives. Commun. Nonlinear Sci. Numer. Simul. 2014, 19, 2354-2372. [CrossRef]

39. Stefanini, L. A generalization of Hukuhara difference and division for interval and fuzzy arithmetic. Fuzzy Sets Syst. 2010, 161, 1564-1584. [CrossRef]

40. Zadeh, L.A. Fuzzy sets. Inf. Control 1965, 8, 338-353. [CrossRef]

41. Osuna-Gomez, R.; Jimenez-Gamero, M.D.; Chalco-Cano, Y.; Rojas-Medar, M.A. Hadamard and Jensen Inequalities for s-Convex Fuzzy Processes. In Soft Methodology and Random Information Systems; Advances in Soft Computing; Springer: Berlin/Heidelberg, Germany, 2004; Volume 26, pp. 1-15.

42. Costa, T.M. Jensen's inequality type integral for fuzzy-interval-valued functions. Fuzzy Sets Syst. 2017, 327, 31-47. [CrossRef]

43. Kulish, U.; Miranker, W. Computer Arithmetic in Theory and Practice; Academic Press: New York, NY, USA, 2014.

44. Zhao, D.F.; An, T.Q.; Ye, G.J.; Liu, W. New Jensen and Hermite-Hadamard type inequalities for h-convex interval-valued functions. J. Inequal. Appl. 2018, 2018, 302. [CrossRef]

45. Costa, T.M.; Roman-Flores, H. Some integral inequalities for fuzzy-interval-valued functions. Inf. Sci. 2017, 420, 110-125. [CrossRef]

46. Breckner, W.W. Stetigkeitsaussagen für eine Klasse verallgemeinerter konvexer funktionen in topologischen linearen Räumen. Pupl. Inst. Math. 1978, 23, 13-20.

47. SDragomir, S.; Pecaric, J.; Persson, L.E. Some inequalities of Hadamard type. Soochow J. Math. 1995, $21,335-341$.

48. Fejer, L. Uberdie Fourierreihen, II. Math. Naturwiss Anz. Ungar. Akad. Wiss. 1906, 24, 369-390.

49. Kunt, M.; İşcan, İ. Hermite-Hadamard-Fejer type inequalities for p-convex functions. Arab. J. Math. Sci. 2017, 23, 215-230. [CrossRef]

50. Kaleva, O. Fuzzy differential equations. Fuzzy Sets Syst. 1987, 24, 301-317. [CrossRef]

51. Khan, M.B.; Noor, M.A.; Noor, K.I.; Nisar, K.S.; Ismail, K.A.; Elfasakhany, A. Some Inequalities for LR- $\$ \$ \backslash$ left $\left(\{\mathrm{h}\} \_\{1\},\{\mathrm{h}\} \_\{2\} \backslash\right.$ right $)$ \$\$ h 1, h 2-Convex Interval-Valued Functions by Means of Pseudo Order Relation. Int. J. Comput. Intell. Syst. 2021, 14, 1-15. [CrossRef]

52. Sana, G.; Khan, M.B.; Noor, M.A.; Mohammed, P.O.; Chu, Y.M. Harmonically convex fuzzy-interval-valued functions and fuzzy-interval Riemann-Liouville fractional integral inequalities. Int. J. Comput. Intell. Syst. 2021, 14, 1809-1822. [CrossRef]

53. Khan, M.B.; Noor, M.A.; Al-Bayatti, H.M.; Noor, K.I. Some new inequalities for LR-log-h-convex interval-valued functions by means of pseudo order relation. Appl. Math. Inf. Sci. 2021, 15, 459-470.

54. Khan, M.B.; Mohammed, P.O.; Noor, M.A.; Hamed, Y.S. New Hermite-Hadamard inequalities in fuzzy-interval fractional calculus and related inequalities. Symmetry 2021, 13, 673. [CrossRef] 
55. Guo, Y.; Ye, G.; Liu, W.; Zhao, D.; Treanţă, S. Optimality conditions and duality for a class of generalized convex interval-valued optimization problems. Mathematics 2021, 9, 2979. [CrossRef]

56. Treanţă, S. Characterization results of solutions in interval-valued optimization problems with mixed constraints. J. Glob. Optim. 2020, 2020, 1-14. [CrossRef]

57. JMacías-Díaz, E.; Khan, M.B.; Noor, M.A.; Allah, A.M.A.; Alghamdi, S.M. Hermite-Hadamard inequalities for generalized convex functions in interval-valued calculus. AIMS Math. 2022, 7, 4266-4292. [CrossRef]

58. Khan, M.B.; Noor, M.A.; Al-Shomrani, M.M.; Abdullah, L. Some Novel Inequalities for LR-h-Convex Interval-Valued Functions by Means of Pseudo Order Relation. Math. Methods Appl. Sci. 2022, 45, 1310-1410. [CrossRef]

59. Khan, M.B.; Noor, M.A.; Abdeljawad, T.; Abdalla, B.; Althobaiti, A. Some fuzzy-interval integral inequalities for harmonically convex fuzzy-interval-valued functions. AIMS Math. 2022, 7, 349-370. [CrossRef]

60. Mesiar, R.; Li, J.; Pap, E. The Choquet integral as Lebesgue integral and related inequalities. Kybernetika 2010, 46, $1098-1107$.

61. Agahi, H.; Mesiar, R.; Ouyang, Y.; Pap, E.; Štrboja, M. Berwald type inequality for Sugeno integral. Appl. Math. Comput. 2010, 217, 4100-4108. [CrossRef] 